\title{
Discretisation of abstract linear evolution equations of parabolic type
}

Fernando F Gonçalves ${ }^{1,2^{*}}$, Maria do Rosário Grossinho $0^{1,2}$ and Eva Morais ${ }^{1,3}$

\author{
* Correspondence: \\ fgoncalves@iseg.utl.pt \\ ${ }^{1}$ CEMAPRE, ISEG - Technical \\ University of Lisbon, Rua do \\ Quelhas 6, 1200-781 Lisboa, \\ Portugal \\ Full list of author information is \\ available at the end of the article
}

\begin{abstract}
We investigate the discretisation of the linear parabolic equation $d u / d t=A(t) u+f(t)$ in abstract spaces, making use of both the implicit and the explicit finite-difference schemes. The stability of the explicit scheme is obtained, and the schemes' rates of convergence are estimated. Additionally, we study the special cases where $A$ and $f$ are approximated by integral averages and also by weighted arithmetic averages.
\end{abstract}

MSC 2010: 65J10.

Keywords: parabolic evolution equations, finite-difference methods, financial mathematics

\section{Introduction}

In this article, we study the discretisation, with finite-difference methods, of the evolution equation problem

$$
\frac{d u}{d t}=A(t) u+f(t) \text { in }[0, T], \quad u(0)=g,
$$

where, for every $t \in[0, T]$ with $T \in(0, \infty), A(t)$ is a linear operator from a reflexive separable Banach space $V$ to its dual $V^{*}, u:[0, T] \rightarrow V$ is an unknown function, $f:[0$, $T] \rightarrow V^{*}, g$ belongs to a Hilbert space $H$, with $f$ and $g$ given, and $V$ is continuously and densely embedded into $H$. We assume that operator $A(t)$ is continuous and impose a coercivity condition.

Our motivation lies in the numerical approximation of multidimensional PDE problems arising in European financial option pricing. Let us consider the stochastic modeling of a multi-asset financial option of European type under the framework of a general version of Black-Scholes model, where the vector of asset appreciation rates and the volatility matrix are taken time and space-dependent. Owing to a FeynmanKač type formula, pricing this option can be reduced to solving the Cauchy problem (with terminal condition) for a second-order linear parabolic PDE of nondivergent type, with null term and unbounded coefficients, degenerating in the space variables (see, e.g., [1]).

After a change of the time variable, the PDE problem is written

$$
\frac{\partial u}{\partial t}=L u+f \text { in }[0, T] \times \mathbb{R}^{d}, \quad u(0, x)=g(x) \text { in } \mathbb{R}^{d}
$$

(c) 2012 Gonçalves et al; licensee Springer. This is an Open Access article distributed under the terms of the Creative Commons Attribution License (http://creativecommons.org/licenses/by/2.0), which permits unrestricted use, distribution, and reproduction in any medium, provided the original work is properly cited. 
where $L$ is the second-order partial differential operator in the nondivergence form

$$
L(t, x)=a^{i j}(t, x) \frac{\partial^{2}}{\partial x_{i} \partial x_{j}}+b^{i}(t, x) \frac{\partial}{\partial x_{i}}+c(t, x), \quad i, j=1, \ldots, d,
$$

with real coefficients, $f$ and $g$ are given real-valued functions (the free term $f$ is included to further improve generality), and $T \in(0, \infty)$ is a constant. For each $t \in[0$, $T]$ the operator - $L$ is degenerate elliptic, and the growth in the spatial variables of the coefficients $a, b$, and of the free data $f, g$ is allowed. One possible approach for the numerical approximation of the PDE problem (2) is to proceed to a two-stage discretisation. First, the problem is semi-discretised in space, and both the possible equation degeneracy and coefficient unboundedness are dealt with (see, e.g., [2,3], where the spatial approximation is pursued in a variational framework, under the strong assumption that the PDE does not degenerate, and [4]). Subsequently, a time discretisation takes place.

For the time discretisation, the topic of the present article, it can be tackled by approximating the linear evolution equation problem (1) which the PDE problem (2) can be cast into. This simpler general approach, which we follow, is powerful enough to obtain the desired results. On the other hand, it covers a variety of problems, namely initial-value and initial boundary-value problems for linear parabolic PDEs of any order $m \geq 2$.

Several studies dealing with the discretisation of parabolic evolution problems in abstract spaces can be found in the literature. Most of them are concerned with the discretisation of problems with constant operator $A$ (see, e.g., [5-9]). Other studies (see, e.g., [10-13]), study the general case where the operator $A$ is time-dependent, under Hölder or Lipschitz-continuity assumptions. Also, in some of the above mentioned studies and in others, as in [14], the discretisation is pursued by considering a particular discretisation of the datum $f$ (namely, by using integral averages).

In the present study, we study the discretisation in time of problem (1) with timedependent operator $A$ in a general setting. We use both the implicit and the explicit finite-difference schemes. To further improve generality, we proceed to the study leaving the discretised versions of $A$ and $f$ nonspecified. Also, in order to obtain the convergence of the schemes, we need to assume that the solution of (1) satisfies a smoothness condition but weaker than the usual Hölder-continuity.

It is well known that, to guarantee the explicit scheme stability, an additional assumption has to be made, usually involving an inverse inequality between $V$ and $H$ (see, e.g., [15]). In our study, the explicit discretisation is investigated by assuming instead a not usual inverse inequality between $H$ and $V^{* *}$.

In addition, we illustrate our study by exploring examples where different choices are made for the discretised versions of $A$ and $f$.

First, we consider the approximation of $A$ and $f$ by integral averages. We show that the standard smoothness and coercivity assumptions for problem (1) induce correspondent properties for the discretised problem, so that stability results can be proved. Moreover, the rate of convergence we obtain is optimal. Then, we study the alternative approximation of $A$ and $f$ by weighted arithmetic averages of their respective values at consecutive time-grid points. In this case, stronger smoothness assumptions are needed in order to obtain the scheme convergence. 
We emphasize that none of the above mentioned choices is artificial: there are applications where the available information regards the values of $A$ and $f$ at the time-grid points and others the integral averages, but usually not both.

The article is organized as follows. In Section 2, we set an abstract framework for a linear parabolic evolution equation and present a solvability classical result. In the following two sections, we study the discretisation of the evolution equation with the use of the Euler's implicit scheme (Section 3) and the Euler's explicit scheme (Section 4). In Sections 5 and 6, we discuss some examples, respectively, for the implicit and the explicit discretisation schemes and, finally, in Section 7, we present some computational results.

\section{Preliminaries}

We establish some facts on the solvability of linear evolution equations of parabolic type.

Let $V$ be a reflexive separable Banach space embedded continuously and densely into a Hilbert space $H$ with inner product (., .). Then $H^{*}$, the dual space of $H$, is also continuously and densely embedded into $V^{*}$, the dual of $V$. Let us use the notation $\langle\cdot, \cdot\rangle$ for the dualization between $V$ and $V^{*}$. Let $H^{*}$ be identified with $H$ in the usual way, by the Riesz isomorphism. Then we have the so called normal (or Gelfand) triple

$$
V \hookrightarrow H \equiv H^{*} \hookrightarrow V^{*},
$$

with continuous and dense embeddings. It follows that $\langle u, v\rangle=(u, v)$, for all $u \in H$ and for all $v \in V$. Furthermore, $|\langle u, v\rangle| \leq\|u\|_{V^{*}}\|v\|_{\nu}$, for all $u \in V^{* *}$ and for all $v \in V$ (the notation $\|\cdot\|_{X}$ stands for the Banach space $X$ norm). Let us consider the Cauchy problem for an evolution equation

$$
\frac{d u}{d t}=A(t) u+f(t) \text { in }[0, T], \quad u(0)=g,
$$

with $T \in(0, \infty)$, where $A(t)$ is a linear operator from $V$ to $V^{* *}$ for every $t \in[0, T]$ and $A(\cdot) v:[0, T] \rightarrow V^{*}$ is measurable for fixed $v \in V, u:[0, T] \rightarrow V$ is an unknown differentiable function, $f:[0, T] \rightarrow V^{*}$ is a measurable given function, $d / d t$ is the standard derivative with respect to the time variable $t$, and $g \in H$ is given.

We assume that the operator $A(t)$ is continuous and impose a coercivity condition, as well as some regularity on the free data $f$ and $g$.

Assumption 1. Suppose that there exist constants $\lambda>0, K, M$, and $N$ such that

$$
\begin{aligned}
& \text { 1. }\langle A(t) v, v\rangle+\lambda\|v\|_{V}^{2} \leq K\|v\|_{H}^{2}, \forall v \in V \text { and } \forall t \in[0, T] ; \\
& \text { 2. }\|A(t) v\|_{V^{*}} \leq M\|v\|_{V}, \forall v \in V \text { and } \forall t \in[0, T] ; \\
& \text { 3. } \int_{0}^{T}\|f(t)\|_{V^{*}}^{2} d t \leq N \text { and }\|g\|_{H} \leq N .
\end{aligned}
$$

We define the generalized solution of problem (3).

Definition 1. We say that $u \in C([0, T] ; H)$ is a generalized solution of (3) on $[0, T]$ if

$$
\begin{aligned}
& \text { 1. } u \in L^{2}([0, T] ; V) ; \\
& \text { 2. }(u(t), v)=(g, v)+\int_{0}^{t}\langle A(s) u(s), v\rangle d s+\int_{0}^{t}\langle f(s), v\rangle d s, \quad \forall v \in V, \forall t \in[0, T] .
\end{aligned}
$$


Let $X$ be a Banach space with norm $\|\cdot\|_{X}$. We denote by $C([0, T] ; X)$ the space of all continuous $X$-valued functions $z$ on $[0, T]$ such that

$$
\|z\|_{C([0, T], X)}:=\max _{0 \leq t \leq T}\|z(t)\|_{X}<\infty
$$

and by $L^{2}([0, T] ; X)$ the space comprising all strongly measurable functions $w:[0, T]$ $\rightarrow X$ such that

$$
\|w\|_{L^{2}([0, T] ; X)}:=\left(\int_{0}^{T}\|w(t)\|_{X}^{2} d t\right)^{1 / 2}<\infty .
$$

The following well-known result states the existence and uniqueness of the generalized solution of problem (3) (see, e.g., [16]).

Theorem 1. Under conditions (1)-(3) of Assumption 1, problem (3) has a unique generalized solution on $[0, T]$. Moreover

$$
\sup _{t \in[0, T]}\|u(t)\|_{H}^{2}+\int_{0}^{T}\|u(t)\|_{V}^{2} d t \leq N\left(\|g\|_{H}^{2}+\int_{0}^{T}\|f(t)\|_{V^{*}}^{2} d t\right),
$$

where $N$ is a constant.

\section{Implicit discretisation}

We will now study the time discretisation of problem (3) making use of an implicit finite-difference scheme. We begin by constructing an appropriate discrete framework.

Take a number $T \in(0, \infty)$, a non-negative integer $n$ such that $T / n \in(0,1]$, and define the $n$-grid on $[0, T]$

$$
T_{n}=\{t \in[0, T]: t=j k, \quad j=0,1, \ldots, n\},
$$

where $k:=T / n$. Denote $t_{j}=j k$ for $j=0,1, \ldots, n$.

For all $z \in V$, we consider the backward difference quotient

$$
\Delta^{-} z\left(t_{j+1}\right)=k^{-1}\left(z\left(t_{j+1}\right)-z\left(t_{j}\right)\right), \quad j=0,1, \ldots, n-1 .
$$

Let $A_{k}, f_{k}$ be some time-discrete versions of $A$ and $f$, respectively, i.e., $A_{k}\left(t_{j}\right)$ is a linear operator from $V$ to $V^{*}$ for every $j=0,1, \ldots, n$ and $f_{k}: T_{n} \rightarrow V^{*}$ a function. For all $z \in V$, denote $A_{k, j+1} z=A_{k}\left(t_{j+1}\right)_{z}, f_{k, j+1}=f_{k}\left(t_{j+1}\right), j=0,1, \ldots, n-1$.

For each $n \geq 1$ fixed, we define $v_{j}=v\left(t_{j}\right), j=0,1, \ldots, n$, a vector in $V$ satisfying

$$
\Delta^{-} v_{i+1}=A_{k, i+1} v_{i+1}+f_{k, i+1} \text { for } i=0,1, \ldots, n-1, \quad v_{0}=g .
$$

Problem (5) is a time-discrete version of problem (3).

\section{Assumption 2. Suppose that}

$$
\begin{aligned}
& \text { 1. }\left\langle A_{k, j+1} v, v\right\rangle+\lambda\|v\|_{V}^{2} \leq K\|v\|_{H}^{2}, \forall v \in V, j=0,1, \ldots, n-1, \\
& \text { 2. }\left\|A_{k, j+1} v\right\|_{V^{*}} \leq M\|v\|_{V}, \forall v \in V, j=0,1, \ldots, n-1, \\
& \text { 3. } \sum_{j=0}^{n-1}\left\|f_{k, j+1}\right\|_{V^{*}}^{2} k \leq N \text { and }\|g\|_{H} \leq N,
\end{aligned}
$$


where $\lambda, K, M$, and $N$ are the constants in Assumption 1.

Remark 1. Note that as problem (5) is a time-discrete version of problem (3) and $g$ denotes the same function in both problems, under Assumption 1 we have that $g \in H$ and $\|g\|_{H} \leq N$.

Under the above assumption, we establish the existence and uniqueness of the solution of problem (5).

Theorem 2. Let Assumption 2 be satisfied and the constant $K$ be such that $K k \leq 1$. Then for all $n \in \mathbb{N}$ there exists a unique vector $v_{0}, v_{1}, \ldots, v_{n}$ in $V$ satisfying (5).

To prove this result, we consider the following well known lemma (see, e.g., [16,17]).

Lemma 1 (Lax-Milgram). Let $B: V \rightarrow V^{*}$ be a bounded linear operator. Assume there exists $\lambda>0$ such that $\langle B v, v\rangle \geq \lambda\|v\|_{V}^{2}$, for all $v \in V$. Then $B v=v^{*}$ has a unique solution $v \in V$ for every given $v^{*} \in V^{*}$.

Proof. (Theorem 2)

From (5), we have that $\left(I-k A_{k, 1}\right) v_{1}=g+f_{k, 1} k$ and

$\left(I-k A_{k, i+1}\right) v_{i+1}=v_{i}+f_{k, i+1} k$, for $i=0,1, \ldots, n-1$, with $I$ the identity operator on $V$.

We first check that the operators $I-k A_{k, j+1}, j=0,1, \ldots, n-1$, satisfy the hypotheses of Lemma 1. These operators are obviously bounded. We have to show that there exists $\lambda>0$ such that $\left\langle\left(I-k A_{k, j+1}\right) v, v\right\rangle \geq \lambda\|v\|_{V}^{2}$, for all $v \in V, j=0,1, \ldots, n-1$. Owing to (1) in Assumption 2, we have

$$
\begin{aligned}
\left\langle\left(I-k A_{k, j+1}\right) v, v\right\rangle & =\left\langle I v-k A_{k, j+1} v, v\right\rangle=\|v\|_{H}^{2}-k\left\langle A_{k, j+1} v, v\right\rangle \\
& \geq\|v\|_{H}^{2}-k K\|v\|_{H}^{2}+k \lambda\|v\|_{V}^{2} .
\end{aligned}
$$

Then, as $K k \leq 1$, we have that $\left\langle\left(I-k A_{k, j+1}\right) v, v\right\rangle \geq k \lambda\|v\|_{V}^{2}$ and the hypotheses of Lemma 1 are satisfied.

For $v_{1}$, we have that $\left(I-k A_{k 1}\right) v_{1}=g+f_{k, 1} k$. This equation has a unique solution by Lemma 1. Suppose now that equation $\left(I-k A_{k, i}\right) v_{i}=v_{i-1}+f_{k, i} k$ has a unique solution. Then equation $\left(I-k A_{k, i+1}\right) v_{i+1}=v_{i}+f_{k, i+1} k$ has also a unique solution, again by Lemma 1 . The result is obtained by induction.

Next, we prove an auxiliary result and then obtain a version of the discrete Gronwall's lemma convenient for our purposes.

Lemma 2. Let $a_{1}^{n}, a_{2}^{n}, \ldots, a_{n}^{n}$ be a finite sequence of numbers for every integer $n \geq 1$ such that $0 \leq a_{j}^{n} \leq c_{0}+C \sum_{i=1}^{j-1} a_{i}^{n}$, for all $j=1,2, \ldots, n$, where $C$ is a positive constant and $c_{0}$ $\geq 0$ is some real number. Then $a_{j}^{n} \leq(C+1)^{j-1} c_{0}$, for all $j=1,2, \ldots, n$.

Proof. Let $b_{j}^{n}:=c_{0}+C \sum_{i=1}^{j-1} b_{i}^{n}, j=1,2, \ldots, n$. Then $a_{j}^{n} \leq b_{j}^{n}$ for all $j \geq 1$. Indeed for $j=$ 1 , we have that $a_{1}^{n} \leq b_{1}^{n}=c_{0}$. Assume now that $a_{i}^{n} \leq b_{i}^{n}$ for all $i \leq j$. Then

$$
b_{j+1}^{n}=c_{0}+C \sum_{i=1}^{j} b_{i}^{n} \geq c_{0}+C \sum_{i=1}^{j} a_{i}^{n} \geq a_{j+1}^{n}
$$

and, by induction, $a_{j}^{n} \leq b_{j}^{n}$ for all $j \geq 1$. It is easy to see that $b_{j+1}^{n}-b_{j}^{n}=C b_{j}^{n}, j \geq 1$, giving

$$
a_{j+1}^{n} \leq b_{j+1}^{n}=(C+1) b_{j}^{n}=(C+1)^{2} b_{j-1}^{n}=\ldots=(C+1)^{j} b_{1}^{n}=(C+1)^{j} c_{0},
$$

and the result is proved. 
Lemma 3 (Discrete Gronwall's inequality). Let $a_{0}^{n}, a_{1}^{n}, \ldots, a_{n}^{n}$ be a finite sequence of numbers for every integer $n \geq 1$ such that

$$
0 \leq a_{j}^{n} \leq a_{0}^{n}+K \sum_{i=1}^{j} a_{i}^{n} k
$$

holds for every $j=1,2, \ldots, n$, with $k:=T / n$, and $K$ a positive number such that $K k=$ : $q<1$, with $q$ a fixed constant. Then

$$
a_{j}^{n} \leq a_{0}^{n} e^{K_{q} T}
$$

for all integers $n \geq 1$ and $j=1,2, \ldots, n$, where $K_{q}:=-K \ln (1-q) / q$.

Proof. The result is obtained by using standard discrete Gronwall arguments. From (6), as $K k<1$ we have

$$
(1-K k) a_{j}^{n} \leq a_{0}^{n}+K \sum_{i=1}^{j-1} a_{i}^{n} k \Leftrightarrow a_{j}^{n} \leq \frac{a_{0}^{n}}{1-K k}+\frac{K k}{1-K k} \sum_{i=1}^{j-1} a_{i}^{n},
$$

for every $j=1,2, \ldots, n$. Owing to Lemma 2 , with $c_{0}=a_{0}^{n} /(1-K k)$ and $C=K k /(1-$ $K k$ ), from the right inequality in (7) we obtain

$$
a_{j}^{n} \leq\left(\frac{K k}{1-K k}+1\right)^{j-1} \frac{a_{0}^{n}}{1-K k}=\frac{a_{0}^{n}}{(1-K k)^{j}} \leq \frac{a_{0}^{n}}{(1-K k)^{n}} .
$$

Noting that

$$
\begin{aligned}
(1-K k)^{n} & =\exp (n \ln (1-K k))=\exp \left(n K k \frac{\ln (1-q)}{q}\right) \\
& =\exp \left(K T \frac{\ln (1-q)}{q}\right),
\end{aligned}
$$

the result is proved.

We are now able to prove that the scheme (5) is stable, that is, the solution of the discrete problem remains bounded independently of $k$.

Theorem 3. Let Assumption 2 be satisfied and assume further that constant $K$ satisfies: $2 K k<1$. Denote $v_{k, j}$, with $j=0,1, \ldots, n$, the unique solution of problem (5) in Theorem 2. Then there exists a constant $N$ independent of $k$ such that

$$
\begin{aligned}
& \text { 1. } \max _{0 \leq j \leq n}\left\|v_{k, j}\right\|_{H}^{2} \leq N\left(\|g\|_{H}^{2}+\sum_{j=1}^{n}\left\|f_{k, j}\right\|_{V^{*}}^{2} k\right) ; \\
& \text { 2. } \sum_{j=0}^{n}\left\|v_{k, j}\right\|_{V}^{2} k \leq N\left(\|g\|_{H}^{2}+\sum_{j=1}^{n}\left\|f_{k, j}\right\|_{V^{*}}^{2} k\right) .
\end{aligned}
$$

Remark 2. Owing to (3) in Assumption 2, the estimates (1) and (2) above can be written, respectively,

$$
\sup _{n \geq 1}\left(\max _{0 \leq j \leq n}\left\|v_{k, j}\right\|_{H}^{2} \leq N\right) \quad \text { and } \sup _{n \geq 1}\left(\sum_{j=0}^{n}\left\|v_{k, j}\right\|_{V}^{2} k \leq N\right) .
$$


Remark 3. Under Assumption 2, with $K$ satisfying $2 K k<1$, Theorem 2 obviously holds so that problem (5) has a unique solution.

Proof. (Theorem 3)

For $i=0,1, \ldots, n-1$, we have that

$$
\left\|v_{k, i+1}\right\|_{H}^{2}-\left\|v_{k, i}\right\|_{H}^{2}=2\left\langle v_{k, i+1}-v_{k, i}, v_{k, i+1}\right\rangle-\left\|v_{k, i+1}-v_{k, i}\right\|_{H}^{2}
$$

and, summing up both members of equation (8), we obtain, for $j=1,2, \ldots, n$,

$$
\left\|v_{k, j}\right\|_{H}^{2}=\left\|v_{k, 0}\right\|_{H}^{2}+\sum_{i=0}^{j-1} 2\left\langle v_{k, i+1}-v_{k, i}, v_{k, i+1}\right\rangle-\sum_{i=0}^{j-1}\left\|v_{k, i+1}-v_{k, i}\right\|_{H}^{2} .
$$

Hence

$$
\begin{aligned}
\left\|v_{k, j}\right\|_{H}^{2} & \leq\left\|v_{k, 0}\right\|_{H}^{2}+\sum_{i=0}^{j-1} 2\left\langle v_{k, i+1}-v_{k, i}, v_{k, i+1}\right\rangle \\
& =\left\|v_{k, 0}\right\|_{H}^{2}+\sum_{i=0}^{j-1} 2\left\langle A_{k, i+1} v_{k, i+1} k+f_{k, i+1} k, v_{k, i+1}\right\rangle .
\end{aligned}
$$

As, by Cauchy's inequality,

$$
2\left\langle f_{k, i+1}, v_{k, i+1}\right\rangle k \leq \lambda\left\|v_{k, i+1}\right\|_{V}^{2} k+\frac{1}{\lambda}\left\|f_{k, i+1}\right\|_{V^{*}}^{2} k,
$$

with $\lambda>0$, owing to (1) in Assumption 2 we obtain

$$
\left\|v_{k, j}\right\|_{H}^{2} \leq\left\|v_{k, 0}\right\|_{H}^{2}+2 K \sum_{i=0}^{j-1}\left\|v_{k, i+1}\right\|_{H}^{2} k-\lambda \sum_{i=0}^{j-1}\left\|v_{k, i+1}\right\|_{H}^{2} k+\frac{1}{\lambda} \sum_{i=0}^{j-1}\left\|f_{k, i+1}\right\|_{V^{*}}^{2} k,
$$

and then

$$
\left\|v_{k, j}\right\|_{H}^{2}+\lambda \sum_{i=1}^{j}\left\|v_{k, i}\right\|_{V}^{2} k \leq\left\|v_{k, 0}\right\|_{H}^{2}+2 K \sum_{i=1}^{j}\left\|v_{k, i}\right\|_{H}^{2} k+\frac{1}{\lambda} \sum_{i=1}^{n}\left\|f_{k, i}\right\|_{V^{*}}^{2} k .
$$

In particular,

$$
\left\|v_{k, j}\right\|_{H}^{2} \leq\left\|v_{k, 0}\right\|_{H}^{2}+2 K \sum_{i=1}^{j}\left\|v_{k, i}\right\|_{H}^{2} k+\frac{1}{\lambda} \sum_{i=1}^{n}\left\|f_{k, i}\right\|_{V^{*}}^{2} k,
$$

and, using Lemma 3,

$$
\left\|v_{k, j}\right\|_{H}^{2} \leq\left(\left\|v_{k, 0}\right\|_{H}^{2}+\frac{1}{\lambda} \sum_{i=1}^{n}\left\|f_{k, i}\right\|_{V^{*}}^{2} k\right) e^{2 K_{q} T},
$$

where $K_{q}$ is the constant defined in the Lemma. Estimate (1) follows. From (9), (10), and (11) we finally obtain

$$
\left\|v_{k, j}\right\|_{H}^{2}+\lambda \sum_{i=1}^{j}\left\|v_{k, i}\right\|_{V}^{2} k \leq\left(\left\|v_{k, 0}\right\|_{H}^{2}+\frac{1}{\lambda} \sum_{i=1}^{n}\left\|f_{k, i}\right\|_{V^{*}}^{2} k\right) e^{2 K_{q} T}
$$


and

$$
\sum_{i=1}^{j}\left\|v_{k, i}\right\|_{V}^{2} k \leq\left(\left\|v_{k, 0}\right\|_{H}^{2}+\frac{1}{\lambda} \sum_{i=1}^{n}\left\|f_{k, i}\right\|_{V^{*}}^{2} k\right) \frac{1}{\lambda} e^{2 K_{q} T} .
$$

Estimate (2) follows.

We will now study the convergence properties of the scheme we have constructed. We impose stronger regularity on the solution $u=u(t)$ of problem (3):

Assumption 3. Let $u$ be the solution of problem (3) in Theorem 1. We suppose that there exist a fixed number $\delta \in(0,1]$ and a constant $C$ such that

$$
\frac{1}{k} \int_{t_{i}}^{t_{i+1}}\left\|u\left(t_{i+1}\right)-u(s)\right\|_{V} d s \leq C k^{\delta}
$$

for all $i=0,1, \ldots, n-1$.

Remark 4. Assume that $u$ satisfies the following condition: "There exist a fixed number $\delta \in(0,1]$ and a constant $C$ such that $\|u(t)-u(s)\|_{V} \leq C|t-s|^{\delta}$, for all $s, t \in[0, T]$ ". Then Assumption 3 obviously holds.

By assuming this stronger regularity of the solution $u$ of (3), we can prove the convergence of the solution of problem (5) to the solution of problem (3) and determine the convergence rate. The accuracy we obtain is of order $\delta$.

Theorem 4. Let Assumptions 1 and 2 be satisfied and assume further that constant $K$ satisfies: $2 K k<1$. Denote $u(t)$ the unique solution of (3) in Theorem 1 and $v_{k, j}, j=0,1$, ..., $n$, the unique solution of (5) in Theorem 2. Let also Assumption 3 be satisfied. Then there exists a constant $N$ independent of $k$ such that

$$
\begin{aligned}
& \begin{aligned}
\max _{0 \leq j \leq n}\left\|v_{k, j}-u\left(t_{j}\right)\right\|_{H}^{2} \leq & N\left(k^{2 \delta}+\sum_{j=1}^{n} \frac{1}{k}\left\|A_{k, j} u\left(t_{j}\right) k-\int_{t_{j-1}}^{t_{j}} A(s) u\left(t_{j}\right) d s\right\|_{V^{*}}^{2}\right. \\
& \left.+\sum_{j=1}^{n} \frac{1}{k}\left\|f_{k, j} k-\int_{t_{j-1}}^{t_{j}} f(s) d s\right\|_{V^{*}}^{2}\right) ; \\
\sum_{j=0}^{n}\left\|v_{k, j}-u\left(t_{j}\right)\right\|_{V}^{2} k \leq & N\left(k^{2 \delta}+\sum_{j=1}^{n} \frac{1}{k}\left\|A_{k, j} u\left(t_{j}\right) k-\int_{t_{j-1}}^{t_{j}} A(s) u\left(t_{j}\right) d s\right\|_{V^{*}}^{2}\right. \\
& \left.+\sum_{j=1}^{n} \frac{1}{k}\left\|f_{k_{k j}} k-\int_{t_{j-1}}^{t_{j}} f(s) d s\right\|_{V^{*}}^{2}\right)
\end{aligned}
\end{aligned}
$$

Proof. Define $w\left(t_{i}\right):=v_{k, i}-u\left(t_{i}\right), i=0,1, \ldots, n$. For $i=0,1, \ldots, n-1$,

$$
\begin{aligned}
w\left(t_{i+1}\right)-w\left(t_{i}\right) & =A_{k, i+1} w\left(t_{i+1}\right) k+f_{k, i+1} k-u\left(t_{i+1}\right)+u\left(t_{i}\right)+A_{k, i+1} u\left(t_{i+1}\right) k \\
& =A_{k, i+1} w\left(t_{i+1}\right) k+\varphi\left(t_{i+1}\right),
\end{aligned}
$$

where $\phi\left(t_{i+1}\right):=f_{k, i+1} k-u\left(t_{i+1}\right)+u\left(t_{i}\right)+A_{k, i+1} u\left(t_{i+1}\right) k$. 
Owing to (1) in Assumption 2, we obtain

$$
\begin{aligned}
\left\|w\left(t_{i+1}\right)\right\|_{H}^{2}-\left\|w\left(t_{i}\right)\right\|_{H}^{2}= & 2\left\langle w\left(t_{i+1}\right)-w\left(t_{i}\right), w\left(t_{i+1}\right)\right\rangle-\left\|w\left(t_{i+1}\right)-w\left(t_{i}\right)\right\|_{H}^{2} \\
\leq & 2\left\langle A_{k, i+1} w\left(t_{i+1}\right), w\left(t_{i+1}\right)\right\rangle k+2\left\langle\varphi\left(t_{i+1}\right), w\left(t_{i+1}\right)\right\rangle \\
\leq & -2 \lambda\left\|w\left(t_{i+1}\right)\right\|_{V}^{2} k+2 K\left\|w\left(t_{i+1}\right)\right\|_{H}^{2} k \\
& +2\left|\left\langle\varphi\left(t_{i+1}\right), w\left(t_{i+1}\right)\right\rangle\right| .
\end{aligned}
$$

Noting that $\phi\left(t_{i+1}\right)$ can be written

$$
\varphi\left(t_{i+1}\right)=\int_{t_{i}}^{t_{i+1}} A(s)\left(u\left(t_{i+1}\right)-u(s)\right) d s+\varphi_{1}\left(t_{i+1}\right)+\varphi_{2}\left(t_{i+1}\right),
$$

where

$$
\varphi_{1}\left(t_{i+1}\right):=A_{k, i+1} u\left(t_{i+1}\right) k-\int_{t_{i}}^{t_{i+1}} A(s) u\left(t_{i+1}\right) d s
$$

and

$$
\varphi_{2}\left(t_{i+1}\right):=f_{k, i+1} k-\int_{t_{i}}^{t_{i+1}} f(s) d s,
$$

for the last term in (12) we have the estimate

$$
\begin{aligned}
2\left|\left\langle\varphi\left(t_{i+1}\right), w\left(t_{i+1}\right)\right\rangle\right| \leq 2 & \left|\left\langle\int_{t_{i}}^{t_{i+1}} A(s)\left(u\left(t_{i+1}\right)-u(s)\right) d s, w\left(t_{i+1}\right)\right\rangle\right| \\
& +2\left|\left\langle\varphi_{1}\left(t_{i+1}\right), w\left(t_{i+1}\right)\right\rangle\right|+2\left|\left\langle\varphi_{2}\left(t_{i+1}\right), w\left(t_{i+1}\right)\right\rangle\right| .
\end{aligned}
$$

Let us estimate separately each one of the three terms in (13).

For the first term, owing to (2) in Assumption 1 and using Cauchy's inequality, we obtain

$$
\begin{aligned}
& 2\left|\left\langle\int_{t_{i}}^{t_{i+1}} A(s)\left(u\left(t_{i+1}\right)-u(s)\right) d s, w\left(t_{i+1}\right)\right\rangle\right| \\
& \quad \leq 2 \int_{t_{i}}^{t_{i+1}}\left|\left\langle A(s)\left(u\left(t_{i+1}\right)-u(s)\right) d s, w\left(t_{i+1}\right)\right\rangle\right| d s \\
& \quad \leq 2 M\left\|w\left(t_{i+1}\right)\right\|_{V} \int_{t_{i}}^{t_{i+1}}\left\|u\left(t_{i+1}\right)-u(s)\right\|_{V} d s \\
& \quad \leq \frac{\lambda}{3}\left\|w\left(t_{i+1}\right)\right\|_{V}^{2} k+\frac{3 M^{2}}{\lambda k}\left(\int_{t_{i}}^{t_{i+1}}\left\|u\left(t_{i+1}\right)-u(s)\right\|_{V} d s\right)^{2},
\end{aligned}
$$

with $\lambda>0$. 
For the two remaining terms, we have the estimates

$$
2\left|\left\langle\varphi_{1}\left(t_{i+1}\right), w\left(t_{i+1}\right)\right\rangle\right| \leq \frac{\lambda}{3}\left\|w\left(t_{i+1}\right)\right\|_{V}^{2} k+\frac{3}{\lambda k}\left\|\varphi_{1}\left(t_{i+1}\right)\right\|_{V^{*}}^{2}
$$

and

$$
2\left|\left\langle\varphi_{2}\left(t_{i+1}\right), w\left(t_{i+1}\right)\right\rangle\right| \leq \frac{\lambda}{3}\left\|w\left(t_{i+1}\right)\right\|_{V}^{2} k+\frac{3}{\lambda k}\left\|\varphi_{2}\left(t_{i+1}\right)\right\|_{V^{*}}^{2},
$$

with $\lambda>0$, using Cauchy's inequality.

Therefore, from (14), (15), and (16) we get the following estimate for (13)

$$
\begin{aligned}
2\left|\left\langle\varphi\left(t_{i+1}\right), w\left(t_{i+1}\right)\right)\right| \leq & \lambda\left\|w\left(t_{i+1}\right)\right\|_{V}^{2} k+\frac{3 M^{2}}{\lambda k}\left(\int_{t_{i}}^{t_{i+1}}\left\|u\left(t_{i+1}\right)-u(s)\right\|_{V} d s\right)^{2} \\
& +\frac{3}{\lambda k}\left\|\varphi_{1}\left(t_{i+1}\right)\right\|_{V^{*}}^{2}+\frac{3}{\lambda k}\left\|\varphi_{2}\left(t_{i+1}\right)\right\|_{V^{*}}^{2}
\end{aligned}
$$

Putting estimates (12) and (17) together and summing up, owing to Assumption 3 we obtain, for $j=1,2, \ldots, n$,

$$
\begin{gathered}
\left\|w\left(t_{j}\right)\right\|_{H}^{2}+\lambda \sum_{i=0}^{j-1}\left\|w\left(t_{i+1}\right)\right\|_{V^{2}}^{2} k \leq 2 K \sum_{i=0}^{j-1}\left\|w\left(t_{i+1}\right)\right\|_{H}^{2} k+\frac{3 C^{2} M^{2}}{\lambda} \sum_{i=0}^{j-1} k^{2 \delta+1} \\
+\frac{3}{\lambda k} \sum_{i=0}^{j-1}\left\|\varphi_{1}\left(t_{i+1}\right)\right\|_{V^{*}}^{2}+\frac{3}{\lambda k} \sum_{i=0}^{j-1}\left\|\varphi_{2}\left(t_{i+1}\right)\right\|_{V^{*}}^{2} .
\end{gathered}
$$

Hence

$$
\begin{aligned}
\left\|w\left(t_{j}\right)\right\|_{H}^{2}+\lambda \sum_{i=0}^{j}\left\|w\left(t_{i}\right)\right\|_{V}^{2} k \leq & 2 K \sum_{i=0}^{j}\left\|w\left(t_{i}\right)\right\|_{H}^{2} k+N k^{2 \delta} \\
& +N \sum_{i=1}^{n} \frac{1}{k}\left\|A_{k, i} u\left(t_{i}\right) k-\int_{t_{i-1}}^{t_{i}} A(s) u\left(t_{i}\right) d s\right\|_{V^{*}}^{2} \\
& +N \sum_{i=1}^{n} \frac{1}{k}\left\|f_{k, i} k \int_{t_{i-1}}^{t_{i}} f(s) d s\right\|_{V^{*}}^{2},
\end{aligned}
$$

with $N$ a constant. Following the same steps as in the proof of Theorem 3, estimates (1) and (2) follow.

Next result is an immediate consequence of Theorem 4.

Corollary 1. Let the hypotheses of Theorem 4 be satisfied and denote $u(t)$ the unique solution of (3) in Theorem 1 and $v_{k, j}, j=0,1, \ldots, n$, the unique solution of (5) in Theorem 2. If there exists a constant $N^{\prime}$ independent of $k$ such that

$$
\left\|A_{k, j} u\left(t_{j}\right)-\frac{1}{k} \int_{t_{j-1}}^{t_{j}} A(s) u\left(t_{j}\right) d s\right\|_{V^{*}}^{2}+\left\|f_{k, j}-\frac{1}{k} \int_{t_{j-1}}^{t_{j}} f(s) d s\right\|_{V^{*}}^{2} \leq N^{\prime} k^{2 \delta},
$$


for $j=1,2, \ldots, n$, then

$$
\max _{0 \leq j \leq n}\left\|v_{k, j}-u\left(t_{j}\right)\right\|_{H}^{2} \leq N k^{2 \delta} \text { and } \sum_{j=0}^{n}\left\|v_{k, j}-u\left(t_{j}\right)\right\|_{V}^{2} k \leq N k^{2 \delta},
$$

with $N$ be a constant independent of $k$.

\section{Explicit discretisation}

We now approach the time-discretisation with the use of an explicit finite-difference scheme. As in the previous section, we begin by setting a suitable discrete framework and then investigate the stability and convergence properties of the scheme.

Observe that, when using the explicit scheme, a previous "discretisation in space" has to be assumed. Therefore, we will consider the following version of problem (3) in the spaces $V_{h}, H_{h}$, and $V_{h}^{*}$, "space-discrete versions" of $V, H$, and $V^{*}$, respectively,

$$
\frac{d u}{d t}=A_{h}(t) u+f_{h}(t) \text { in }[0, T], \quad u(0)=g_{h},
$$

with $A_{h}(t), f_{h}(t)$, and $g_{h}$ "space-discrete versions" of $A(t), f(t)$, and $g$, and $h \in(0,1]$ a constant. We will use the notation $(\cdot, \cdot)_{h}$ for the inner product in $H_{h}$ and $\langle\cdot, \cdot\rangle_{h}$ for the duality between $V_{h}^{*}$ and $V_{h}$.

Let the time-grid $T_{n}$ as defined in (4). For all $z \in V_{h}$, consider the forward difference quotient in time

$$
\Delta^{+} z\left(t_{j}\right)=k^{-1}\left(z\left(t_{j+1}\right)-z\left(t_{j}\right)\right), \quad j=0,1, \ldots, n-1 .
$$

Let $A_{h k}, f_{h k}$ be some time-discrete versions of $A_{h}$ and $f_{h}$, respectively, and denote, for all $z \in V_{h}$,

$$
A_{h k, j} z=A_{h k}\left(t_{j}\right) z, \quad f_{h k, j}=f_{h k}\left(t_{j}\right),
$$

with $j=0,1, \ldots, n-1$.

For each $n \geq 1$ fixed, we consider the time-discrete version of (18),

$$
\Delta^{+} v_{i}=A_{h k, i} v_{i}+f_{h k, i} \quad \text { for } \quad i=0,1, \ldots, n-1, \quad v_{0}=g_{h},
$$

with $v_{j}=v\left(t_{j}\right), j=0,1, \ldots, n$, in $V_{h}$.

Problem (19) can be solved uniquely by recursion

$$
v_{j}=g_{h}+\sum_{i=0}^{j-1} A_{h k, i} v_{i} k+\sum_{i=0}^{j-1} f_{h k, i} k \text { for } j=1, \ldots, n, \quad v_{0}=g_{h} .
$$

We make some assumptions.

Assumption 4. Suppose that

$$
\begin{aligned}
& \text { 1. }\left\langle A_{h k, j} v, v\right\rangle_{h}+\lambda\|v\|_{V_{h}}^{2} \leq K\|v\|_{H_{h}}^{2}, \forall v \in V_{h}, j=0,1, \ldots, n-1, \\
& \text { 2. }\left\|A_{h k, j} v\right\|_{V_{h}^{*}} \leq M\|v\|_{V_{h}}, \forall v \in V_{h}, j=0,1, \ldots, n-1, \\
& \text { 3. } \sum_{j=0}^{n-1}\left\|f_{h k, j}\right\|_{V_{h}^{*}}^{2} k \leq N \text { and }\left\|g_{h}\right\|_{H_{h}} \leq N,
\end{aligned}
$$


where $\lambda, K, M$, and $N$ are the constants in Assumption 1.

Remark 5. We refer to Remark 1 and note that, under Assumption 1, $g_{h} \in H_{h}$ and $\left\|g_{h}\right\|_{H_{h}} \leq N$.

The following version of the discrete Gronwall's inequality is an immediate consequence of Lemma 3.

Lemma 4. Let $a_{0}^{n}, a_{1}^{n}, \ldots, a_{n}^{n}$ be a finite sequence of numbers for every integer $n \geq 1$ such that

$$
0 \leq a_{j}^{n} \leq a_{0}^{n}+K \sum_{i=0}^{j-1} a_{i}^{n} k,
$$

holds for every $j=0,1, \ldots, n$, with $k:=T / n$ and $K$ a positive number such that $K k=$ : $q<1$, with $q$ a fixed constant. Then

$$
a_{j}^{n} \leq a_{0}^{n} e^{K_{q} T}
$$

for all integers $n \geq 1$ and $j=0,1, \ldots, n$, where $K_{q}:=-K \ln (1-q) / q$.

Proof. From (20), owing to Lemma 3 we have

$$
(1+K k) a_{j}^{n} \leq(1+K k) a_{0}^{n}+K \sum_{i=1}^{j} a_{i}^{n} k \leq(1+K k) a_{0}^{n} e^{K_{q} T},
$$

for $j=1,2, \ldots, n$. The result follows.

In order to obtain stability for the scheme (19) we make an additional assumption, involving an inverse inequality between $H_{h}$ and $V_{h}^{*}$. We note that, for the case of the implicit scheme, there was no such need: the implicit scheme's stability was met unconditionally.

Assumption 5. Suppose that there exists a constant $C_{h}$, dependent of $h$, such that

$$
\|z\|_{H_{h}} \leq C_{h}\|z\|_{V_{h}^{*},} \quad \text { for all } z \in V_{h} .
$$

Remark 6. The usual assumption involves instead an inverse inequality between $V_{h}$ and $H_{h}$ :

$$
\|z\|_{V_{h}} \leq C_{h}\|z\|_{H_{h^{\prime}}} \quad \text { for all } z \in V_{h} .
$$

It can be easily checked that (22) implies (21). In fact, for all $z \in V_{h}, z \neq 0$,

$$
\|z\|_{V_{h}^{*}}=\sup _{\substack{u \in V_{h} \\ u \neq 0}} \frac{\left|(z, u)_{h}\right|}{\|u\|_{V_{h}}} \geq \frac{\left|(z, z)_{h}\right|}{\|z\|_{V_{h}}}=\frac{\|z\|_{H_{h}}^{2}}{\|z\|_{V_{h}}} \geq \frac{\|z\|_{H_{h}}^{2}}{C_{h}\|z\|_{H_{h}}}=\frac{\|z\|_{H_{h}}}{C_{h}},
$$

with the last inequality above due to (22).

Remark 7. Assumption 5 is not void. For example, when the solvability of a multidimensional linear PDE of parabolic type is considered in Sobolev spaces, and its discretised version solvability in discrete counterparts of those spaces (see [3]), (21) is satisfied with $C_{h}$ such that $C_{h}^{2}-1 \geq \mathrm{Ch}^{-2}$, with $C$ a constant independent of $h$.

Theorem 5. Let Assumptions 4 and 5 be satisfied and $\lambda, K, M$, and $C_{h}$ the constants defined in the Assumptions. Denote by $v_{h k, j}$, with $j=0,1, \ldots, n$, the unique solution of problem (19). Assume that constant $K$ is such that $2 K k<1$. If there exists a number $p$ such 
that $M^{2} C_{h}^{2} k \leq p<\lambda$ then there exists a constant $N$, independent of $k$ and $h$, such that

$$
\begin{aligned}
& \text { 1. } \max _{0 \leq j \leq n}\left\|v_{h k, j}\right\|_{H_{h}}^{2} \leq N\left(\left\|g_{h}\right\|_{H_{h}}^{2}+\sum_{j=0}^{n-1}\left\|f_{h k, j}\right\|_{V_{h}^{*}}^{2} k\right) ; \\
& \text { 2. } \sum_{j=0}^{n-1}\left\|v_{h k, j}\right\|_{V_{h}}^{2} k \leq N\left(\left\|g_{h}\right\|_{H_{h}}^{2}+\sum_{j=0}^{n-1}\left\|f_{h k, j}\right\|_{V_{h}^{*}}^{2} k\right) .
\end{aligned}
$$

Remark 8. Remark 2 applies to the above theorem with the obvious adaptations.

Proof. (Theorem 5)

For $i=0,1, \ldots, n-1$, we have

$$
\left\|v_{h k, i+1}\right\|_{H_{h}}^{2}-\left\|v_{h k, i}\right\|_{H_{h}}^{2}=2\left\langle v_{h k, i+1}-v_{h k, i}, v_{h k, i}\right\rangle_{h}+\left\|v_{h k, i+1}-v_{h k, i}\right\|_{H_{h}}^{2}
$$

and, summing up both members of equation (23), for $j=1,2, \ldots, n$, we get

$$
\begin{aligned}
\left\|v_{h k, j}\right\|_{H_{h}}^{2}= & \left\|v_{h k, 0}\right\|_{H_{h}}^{2}+\sum_{i=0}^{j-1} 2\left\langle v_{h k, i+1}-v_{h k, i}, v_{h k, i}\right\rangle_{h}+\sum_{i=0}^{j-1}\left\|v_{h k, i+1}-v_{h k, i}\right\|_{H_{h}}^{2} \\
= & \left\|v_{h k, 0}\right\|_{H_{h}}^{2}+\sum_{i=0}^{j-1} 2\left\langle A_{h k, i} v_{h k, i}, v_{h k, i}\right\rangle_{h} k+\sum_{i=0}^{j-1} 2\left\langle f_{h k, i}, v_{h k, i}\right\rangle_{h} k \\
& +\sum_{i=0}^{j-1}\left\|A_{h k, i} v_{h k, i}+f_{h k, i}\right\|_{H_{h}}^{2} k^{2} .
\end{aligned}
$$

Owing to (1) in Assumption 4 and using Cauchy's inequality, from (24) we obtain the estimate

$$
\begin{gathered}
\left\|v_{h k, j}\right\|_{H_{h}}^{2} \leq\left\|v_{h k, 0}\right\|_{H_{h}}^{2}+2 K \sum_{i=0}^{j-1}\left\|v_{h k, i}\right\|_{H_{h}}^{2} k-\lambda \sum_{i=0}^{j-1}\left\|v_{h k, i}\right\|_{V_{h}}^{2} k \\
+\frac{1}{\lambda} \sum_{i=0}^{j-1}\left\|f_{h k, i}\right\|_{V_{h}^{*}}^{2} k+\sum_{i=0}^{j-1}\left\|A_{h k, i} v_{h k, i}+f_{h k, i}\right\|_{H_{h} k^{2}}^{2}
\end{gathered}
$$

with $\lambda>0$.

For the last term in the above estimate (25), owing to (2) in Assumption 4 and to Assumption 5, and using Cauchy's inequality we obtain

$$
\begin{aligned}
& \sum_{i=0}^{j-1}\left\|A_{h k, i} v_{h k, i}+f_{h k, i}\right\|_{H_{h}}^{2} k^{2} \\
& \quad \leq C_{h}^{2} k \sum_{i=0}^{j-1}\left\|A_{h k, i} v_{h k, i}+f_{h k, i}\right\|_{V_{h}^{*}}^{2} k \\
& \quad \leq(1+\mu) C_{h}^{2} k \sum_{i=0}^{j-1}\left\|A_{h k, i} v_{h k, i}\right\|_{V_{h}^{*}}^{2} k+\left(1+\frac{1}{\mu}\right) C_{h}^{2} k \sum_{i=0}^{j-1}\left\|f_{h k, i}\right\|_{V_{h}^{*}}^{2} k \\
& \quad \leq(1+\mu) M^{2} C_{h}^{2} k \sum_{i=0}^{j-1}\left\|v_{h k, i}\right\|_{V_{h}}^{2} k+\left(1+\frac{1}{\mu}\right) C_{h}^{2} k \sum_{i=0}^{j-1}\left\|f_{h k, i}\right\|_{V_{h}^{*}}^{2} k
\end{aligned}
$$


with $\mu>0$.

Finally, putting estimates (25) and (26) together, we get

$$
\begin{aligned}
\left\|v_{h k, j}\right\|_{H_{h}}^{2} \leq & \left\|v_{h k, 0}\right\|_{H_{h}}^{2}+2 K \sum_{i=0}^{j-1}\left\|v_{h k, i}\right\|_{H_{h}}^{2} k \\
& +\left((1+\mu) M^{2} C_{h}^{2} k-\lambda\right) \sum_{i=0}^{j-1}\left\|v_{h k, i}\right\|_{V_{h}}^{2} k \\
& +\left(\frac{1}{\lambda}+\left(1+\frac{1}{\mu}\right) C_{h}^{2} k\right) \sum_{i=0}^{j-1}\left\|f_{h k, i}\right\|_{V_{h}^{*}}^{2} k .
\end{aligned}
$$

Now, if there is a constant $p$ such that

$$
M^{2} C_{h}^{2} k \leq p \leq \lambda,
$$

implying that, for $\mu$ sufficiently small,

$$
(1+\mu) M^{2} C_{h}^{2} k-\lambda \leq(1+\mu) p-\lambda<0,
$$

then from (27) we obtain the estimate

$$
\begin{aligned}
& \left\|v_{h k, j}\right\|_{H_{h}}^{2}+(\lambda-(1+\mu) p) \sum_{i=0}^{j-1}\left\|v_{h k, i}\right\|_{V_{h}}^{2} k \\
& \leq\left\|v_{h k, 0}\right\|_{H_{h}}^{2}+2 K \sum_{i=0}^{j-1}\left\|v_{h k, i}\right\|_{H_{h}}^{2} k+L \sum_{i=0}^{n-1}\left\|f_{h k, i}\right\|_{V_{h}^{*}}^{2} k^{\prime}
\end{aligned}
$$

where $L:=\left(\mu M^{2}+\lambda(1+\mu) p\right) / \lambda \mu M^{2}$.

In particular,

$$
\left\|v_{h k, j}\right\|_{H_{h}}^{2} \leq\left\|v_{h k, 0}\right\|_{H_{h}}^{2}+2 K \sum_{i=0}^{j-1}\left\|v_{h k, i}\right\|_{H_{h}}^{2} k+L \sum_{i=0}^{n-1}\left\|f_{h k, i}\right\|_{V_{h}^{*}}^{2} k
$$

and, using Lemma 4 ,

$$
\left\|v_{h k, j}\right\|_{H_{h}}^{2} \leq\left(\left\|v_{h k, 0}\right\|_{H_{h}}^{2}+L \sum_{i=0}^{n-1}\left\|f_{h k, i}\right\|_{V_{h}^{*}}^{2} k\right) e^{2 K_{q} T}
$$

where $K_{q}$ is the constant defined in Lemma 4. (1) follows.

From (28), (29), and (30) we finally obtain

$$
\begin{aligned}
\left\|v_{h k, j}\right\|_{H_{h}}^{2}+(\lambda & -(1+\mu) p) \sum_{i=0}^{j-1}\left\|v_{h k, i}\right\|_{V_{h}}^{2} k \infty \\
\leq & \left(\left\|v_{h k, 0}\right\|_{H_{h}}^{2}+L \sum_{i=0}^{n-1}\left\|f_{h k, i}\right\|_{V_{h}^{*}}^{2} k\right) e^{2 K_{q} T}
\end{aligned}
$$

and (2) follows.

Finally, we prove the convergence of the scheme and determine the convergence rate. The accuracy obtained is of order $\delta$, with $\delta$ given by Assumption 3 . 
Theorem 6. Let Assumptions 1, 4, and 5 be satisfied and $\lambda, K, M$, and $C_{h}$ the constants defined in the Assumptions. Denote by $u_{h}(t)$ the unique solution of problem (18) in Theorem 1 and by $v_{h k, j}$, with $j=0,1, \ldots, n$, the unique solution of problem (19). Assume that constant $K$ is such that $2 K k<1$ and that Assumption 3 is satisfied. If there exists a number $p$ such that $M^{2} C_{h}^{2} k \leq p<\lambda$ then there exists a constant $N$, independent of $k$ and $h$, such that

$$
\max _{0 \leq j \leq n}\left\|v_{h k, j}-u_{h}\left(t_{j}\right)\right\|_{H_{h}}^{2} \leq N\left(k^{2 \delta}+\sum_{j=0}^{n-1} \frac{1}{k}\left\|A_{h k, j} u_{h}\left(t_{j}\right) k-\int_{t_{j}}^{t_{j+1}} A_{h}(s) u_{h}\left(t_{j}\right) d s\right\|_{V_{h}^{*}}^{2}\right.
$$

$$
\begin{aligned}
&\left.+\sum_{j=0}^{n-1} \frac{1}{k}\left\|f_{h k, j} k-\int_{t_{j}}^{t_{j+1}} f_{h}(s) d s\right\|_{V_{h}^{*}}^{2}\right) ; \\
& \sum_{j=0}^{n-1}\left\|v_{h k, j}-u_{h}\left(t_{j}\right)\right\|_{V_{h}}^{2} k \leq N\left(k^{2 \delta}+\sum_{j=0}^{n-1} \frac{1}{k}\left\|A_{h k, j} u_{h}\left(t_{j}\right) k-\int_{t_{j}}^{t_{j+1}} A_{h}(s) u_{h}\left(t_{j}\right) d s\right\|_{V_{h}^{*}}^{2}\right.
\end{aligned}
$$

2.

$$
\left.+\sum_{j=0}^{n-1} \frac{1}{k}\left\|f_{h k, j} k-\int_{t_{j}}^{t_{j+1}} f_{h}(s) d s\right\|_{V_{h}^{*}}^{2}\right) \text {. }
$$

Proof. Define $w\left(t_{i}\right):=v_{h k, i}-u_{h}\left(t_{i}\right), i=0,1, \ldots, n$. For $i=0,1, \ldots, n-1$

$$
\begin{aligned}
w\left(t_{i+1}\right)-w\left(t_{i}\right) & =A_{h k, i} w\left(t_{i}\right) k+f_{h k, i} k-u_{h}\left(t_{i+1}\right)+u_{h}\left(t_{i}\right)+A_{h k, i} u_{h}\left(t_{i}\right) k \\
& =A_{h k, i} w\left(t_{i}\right) k+\varphi\left(t_{i}\right)
\end{aligned}
$$

where $\phi\left(t_{i}\right):=f_{h k, i} k-u_{h}\left(t_{i+1}\right)+u_{h}\left(t_{i}\right)+A_{h k, i} u_{h}\left(t_{i}\right) k$.

We have that

$$
\begin{aligned}
\left\|w\left(t_{i+1}\right)\right\|_{H_{h}}^{2}-\left\|w\left(t_{i}\right)\right\|_{H_{h}}^{2}= & 2\left\langle w\left(t_{i+1}\right)-w\left(t_{i}\right), w\left(t_{i}\right)\right\rangle_{h}+\left\|w\left(t_{i+1}\right)-w\left(t_{i}\right)\right\|_{H_{h}}^{2} \\
\leq \leq & 2\left\langle A_{h k, i} w\left(t_{i}\right), w\left(t_{i}\right)\right\rangle_{h} k+2\left|\left\langle\varphi\left(t_{i}\right), w\left(t_{i}\right)\right\rangle_{h}\right| \\
& +\left\|A_{h k, i} w\left(t_{i}\right) k+\varphi\left(t_{i}\right)\right\|_{H_{h}}^{2} .
\end{aligned}
$$

We want to estimate each one of the three terms in (31). For the first term in (31), owing to (1) in Assumption 4, we obtain

$$
2\left\langle A_{h k, i} w\left(t_{i}\right), w\left(t_{i}\right)\right\rangle_{h} k \leq-2 \lambda\left\|w\left(t_{i}\right)\right\|_{V_{h}}^{2} k+2 K\left\|w\left(t_{i}\right)\right\|_{H_{h}}^{2} k .
$$

Noting that $\phi\left(t_{i}\right)$ can be written

$$
\varphi\left(t_{i}\right)=\int_{t_{i}}^{t_{i+1}} A_{h}(s)\left(u_{h}\left(t_{i}\right)-u_{h}(s)\right) d s+\varphi_{1}\left(t_{i}\right)+\varphi_{2}\left(t_{i}\right)
$$

where

$$
\varphi_{1}\left(t_{i}\right):=A_{h k, i} u_{h}\left(t_{i}\right) k-\int_{t_{i}}^{t_{i+1}} A_{h}(s) u_{h}\left(t_{i}\right) d s \quad \text { and } \quad \varphi_{2}\left(t_{i}\right):=f_{h k, i} k-\int_{t_{i}}^{t_{i+1}} f_{h}(s) d s,
$$


for the second term in (31) we have

$$
\begin{aligned}
2\left|\left\langle\varphi\left(t_{i}\right), w\left(t_{i}\right)\right\rangle_{h}\right| \leq 2 & \left|\int_{t_{i}}^{t_{i+1}} A_{h}(s)\left(u_{h}\left(t_{i}\right)-u_{h}(s)\right) d s, w\left(t_{i}\right)\right\rangle \mid \\
& +2\left|\left\langle\varphi_{1}\left(t_{i}\right), w\left(t_{i}\right)\right\rangle_{h}\right|+2\left|\left\langle\varphi_{2}\left(t_{i}\right), w\left(t_{i}\right)\right\rangle_{h}\right|
\end{aligned}
$$

and, following the same steps as in the proof of Theorem 4, we obtain the estimate

$$
\begin{gathered}
2\left|\left\langle\varphi\left(t_{i}\right), w\left(t_{i}\right)\right\rangle_{h}\right| \leq \lambda\left\|w\left(t_{i}\right)\right\|_{V_{h}}^{2} k+\frac{3 M^{2}}{\lambda k}\left(\int_{t_{i}}^{t_{i+1}}\left\|u_{h}\left(t_{i}\right)-u_{h}(s)\right\|_{V_{h}} d s\right)^{2} \\
+\frac{3}{\lambda k}\left\|\varphi_{1}\left(t_{i}\right)\right\|_{V_{h}^{*}}^{2}+\frac{3}{\lambda k}\left\|\varphi_{2}\left(t_{i}\right)\right\|_{V_{h}^{*}}^{2} .
\end{gathered}
$$

Next, we estimate the last term in (31). Owing to (2) in Assumption 4 and to Assumption 5, and using Cauchy's inequality,

$$
\begin{aligned}
\left\|A_{h k, i} w\left(t_{i}\right) k+\varphi\left(t_{i}\right)\right\|_{H_{h}}^{2} & \leq C_{h}^{2}\left\|A_{h k, i} w\left(t_{i}\right) k+\varphi\left(t_{i}\right)\right\|_{V_{h}^{*}}^{2} \\
& \leq(1+\mu) C_{h}^{2}\left\|A_{h k, i} w\left(t_{i}\right)\right\|_{V_{h}^{*}}^{2} k^{2}+\left(1+\frac{1}{\mu}\right) C_{h}^{2}\left\|\varphi\left(t_{i}\right)\right\|_{V_{h}^{*}}^{2} \\
& \leq(1+\mu) M^{2} C_{h}^{2} k\left\|w\left(t_{i}\right)\right\|_{V_{h}}^{2} k+\left(1+\frac{1}{\mu}\right) C_{h}^{2}\left\|\varphi\left(t_{i}\right)\right\|_{V_{h}^{*}}^{2},
\end{aligned}
$$

with $\mu>0$. As, owing to (2) in Assumption 1 and to Cauchy's inequality, $\left\|\varphi\left(t_{i}\right)\right\|_{V_{h}^{*}}^{2}$ in (35) can be estimated by

$$
\begin{aligned}
\left\|\varphi\left(t_{i}\right)\right\|_{V_{h}^{*}}^{2}= & \left\|\int_{t_{i}}^{t_{i+1}} A_{h}(s)\left(u_{h}\left(t_{i}\right)-u_{h}(s)\right) d s+\varphi_{1}\left(t_{i}\right)+\varphi_{2}\left(t_{i}\right)\right\|_{V_{h}^{*}}^{2} \\
\leq & \left(1+v+\frac{1}{v}\right)\left\|\int_{t_{i}}^{t_{i+1}} A_{h}(s)\left(u_{h}\left(t_{i}\right)-u_{h}(s)\right) d s\right\|_{V_{h}^{*}}^{2} \\
& +\left(1+v+\frac{1}{v}\right)\left\|\varphi_{1}\left(t_{i}\right)\right\|_{V_{h}^{*}}^{2}+\left(1+v+\frac{1}{v}\right)\left\|\varphi_{2}\left(t_{i}\right)\right\|_{V_{h}^{*}}^{2} \\
\leq & \left(1+v+\frac{1}{v}\right) M^{2}\left(\int_{t_{i}}^{t_{i+1}}\left\|u_{h}\left(t_{i}\right)-u_{h}(s)\right\|_{V_{h}} d s\right)^{2} \\
& +\left(1+v+\frac{1}{v}\right)\left\|\varphi_{1}\left(t_{i}\right)\right\|_{V_{h}^{*}}^{2}+\left(1+v+\frac{1}{v}\right)\left\|\varphi_{2}\left(t_{i}\right)\right\|_{V_{h}^{*}}^{2}
\end{aligned}
$$

with $v>0$, from (35) and (36), we obtain the following estimate for the last term in (31)

$$
\begin{aligned}
& \left\|A_{h k, i} w\left(t_{i}\right) k+\varphi\left(t_{i}\right)\right\|_{H_{h}}^{2} \leq(1+\mu) M^{2} C_{h}^{2} k\left\|w\left(t_{i}\right)\right\|_{V_{h}}^{2} k \\
& +\left(1+\frac{1}{\mu}\right)\left(1+v+\frac{1}{v}\right) M^{2} C_{h}^{2}\left(\int_{t_{i}}^{t_{i+1}}\left\|u_{h}\left(t_{i}\right)-u_{h}(s)\right\|_{V_{h}} d s\right)^{2} \\
& +\left(1+\frac{1}{\mu}\right)\left(1+v+\frac{1}{v}\right) C_{h}^{2}\left\|\varphi_{1}\left(t_{i}\right)\right\|_{V_{h}^{*}}^{2}+\left(1+\frac{1}{\mu}\right)\left(1+v+\frac{1}{v}\right) C_{h}^{2}\left\|\varphi_{2}\left(t_{i}\right)\right\|_{V_{h}^{*}}^{2} .
\end{aligned}
$$


Putting estimates (32), (34), and (37) together and summing up, owing to Assumption 3, we have, for $j=0,1, \ldots, n$,

$$
\begin{aligned}
\left\|w\left(t_{j}\right)\right\|_{H_{h}}^{2} \leq & 2 K \sum_{i=0}^{j-1}\left\|w\left(t_{i}\right)\right\|_{H_{h}}^{2} k+\left((1+\mu) M^{2} C_{h}^{2} k-\lambda\right) \sum_{i=0}^{j-1}\left\|w\left(t_{i}\right)\right\|_{V_{h}}^{2} k \\
& +M^{2} C^{2}\left(\left(1+\frac{1}{\mu}\right)\left(1+v+\frac{1}{v}\right) C_{h}^{2} k+\frac{3}{\lambda}\right) \sum_{i=0}^{j-1} k^{2 \delta+1} \\
& +\left(\left(1+\frac{1}{\mu}\right)\left(1+v+\frac{1}{v}\right) C_{h k}^{2}+\frac{3}{\lambda}\right) \sum_{i=0}^{j-1} \frac{1}{k}\left\|\varphi_{1}\left(t_{i}\right)\right\|_{V_{h}^{*}}^{2} . \\
& +\left(\left(1+\frac{1}{\mu}\right)\left(1+v+\frac{1}{v}\right) C_{h}^{2} k+\frac{3}{\lambda}\right) \sum_{i=0}^{j-1} \frac{1}{k}\left\|\varphi_{2}\left(t_{i}\right)\right\|_{V_{h}^{*}}^{2} .
\end{aligned}
$$

As we assume that there is a constant $p$ such that

$$
M^{2} C_{h}^{2} k \leq p<\lambda
$$

we have that, for $\mu$ sufficiently small,

$$
(1+\mu) M^{2} C_{h}^{2} k-\lambda \leq(1+\mu) p-\lambda<0 .
$$

Then, from (38),

$$
\begin{aligned}
\left\|w\left(t_{j}\right)\right\|_{H_{h}}^{2}+(\lambda-(1+\mu) p) \sum_{i=0}^{j-1}\left\|w\left(t_{j}\right)\right\|_{V_{h}}^{2} k \\
\leq 2 K \sum_{i=0}^{j-1}\left\|w\left(t_{j}\right)\right\|_{H_{h}}^{2} k+M^{2} C^{2} T L k^{2 \delta} \\
+L \sum_{i=0}^{n-1} \frac{1}{k}\left\|A_{h k, i} u_{h}\left(t_{i}\right) k-\int_{t_{i}}^{t_{i+1}} A_{h}(s) u_{h}\left(t_{i}\right) d s\right\|_{V_{h}^{*}}^{2} \\
+L \sum_{i=0}^{n-1} \frac{1}{k}\left\|f_{h k, i} k-\int_{t_{i}}^{t_{i+1}} f_{h}(s) d s\right\|_{V_{h}^{*}}^{2},
\end{aligned}
$$

where $L:=\left(\left(3 M^{2}+\lambda p+v \lambda p\right) \mu v+\left(1+\mu+v+v^{2}\right) \lambda p\right) / \mu \nu \lambda M^{2}$. Estimates (1) and (2) are obtained following the same steps as in Theorem 5 .

Next result follows immediately from Theorem 6.

Corollary 2. Assume that the hypotheses of Theorem 6 are satisfied. Denote by $u_{h}(t)$

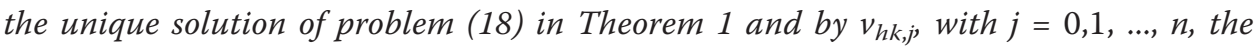
unique solution of problem (19). If there exists a constant $N$, independent of $k$, such that

$$
\left\|A_{h k, j} u_{h}\left(t_{j}\right)-\frac{1}{k} \int_{t_{j}}^{t_{j+1}} A_{h}(s) u_{h}\left(t_{j}\right) d s\right\|_{V_{h}^{*}}^{2}+\left\|f_{h k, j}-\frac{1}{k} \int_{t_{j}}^{t_{j+1}} f_{h}(s) d s\right\|_{V_{h}^{*}}^{2} \leq N^{\prime} k^{2 \delta},
$$


for $j=0,1, \ldots, n-1$, then

$$
\max _{0 \leq j \leq n}\left\|v_{h k, j}-u_{h}\left(t_{j}\right)\right\|_{H_{h}}^{2} \leq N k^{2 \delta} \text { and } \sum_{j=0}^{n-1}\left\|v_{h k, j}-u_{h}\left(t_{j}\right)\right\|_{V_{h}}^{2} k \leq N k^{2 \delta},
$$

with $N$ a constant independent of $k$.

\section{Examples for the implicit scheme}

In this Section, we investigate two possible ways of specifying the discretised operator $A_{k}$ and function $f_{k}$, under the framework of the implicit scheme. We begin by considering the particular case where $A_{k}$ and $f_{k}$ in problem (5) are specified, respectively, by the integral averages

$$
\bar{A}_{k}\left(t_{j+1}\right) z:=\frac{1}{k} \int_{t_{j}}^{t_{j+1}} A(s) z d s \text { and } \bar{f}_{k}\left(t_{j+1}\right):=\frac{1}{k} \int_{t_{j}}^{t_{j+1}} f(s) d s,
$$

for all $z \in V, j=0,1, \ldots, n-1$.

For all $z \in V$, we denote

$$
\bar{A}_{k, j+1} z=\bar{A}_{k}\left(t_{j+1}\right) z, \quad \bar{f}_{k, j+1}=\bar{f}_{k}\left(t_{j+1}\right), \quad j=0,1, \ldots, n-1 .
$$

We prove that, under Assumption 1, $\bar{A}_{k}$ and $\hat{f}_{k}$ satisfy Assumption 2.

Proposition 1. Under Assumption 1, operator $\bar{A}_{k}$ and function $\hat{f}_{k}$ satisfy

$$
\begin{aligned}
& \text { 1. }\left\langle\bar{A}_{k, j+1} v, v\right\rangle+\lambda\|v\|_{V}^{2} \leq K\|v\|_{H}^{2}, \forall v \in V, j=0,1, \ldots, n-1, \\
& \text { 2. }\left\|\bar{A}_{k, j+1} v\right\|_{V^{*}} \leq M\|v\|_{V}, \forall v \in V, j=0,1, \ldots, n-1, \\
& \text { 3. } \sum_{j=0}^{n-1}\left\|\bar{f}_{k, j+1}\right\|_{V^{*}}^{2} k \leq N
\end{aligned}
$$

where $\lambda, K, M$, and $N$ are the constants in Assumption 1.

Proof. For all $v \in V$, owing to (1) in Assumption 1,

$$
\begin{aligned}
\left\langle\bar{A}_{k, j+1} v, v\right\rangle & =\left\langle\frac{1}{k} \int_{t_{j}}^{t_{j+1}} A(s) v d s, v\right\rangle=\frac{1}{k} \int_{t_{j}}^{t_{j+1}}\langle A(s) v, v\rangle d s \\
& \leq \frac{1}{k} \int_{t_{j}}^{t_{j+1}}\left(K\|v\|_{H}^{2}-\lambda\|v\|_{V}^{2}\right) d s \\
& =K\|v\|_{H}^{2}-\lambda\|v\|_{V}^{2},
\end{aligned}
$$

with $j=0,1, \ldots, n-1$, and (1) is proved.

For all $v \in V$, owing to (2) in Assumption 1,

$$
\begin{aligned}
\left\|\bar{A}_{k, j+1} v\right\|_{V^{*}} & =\left\|\frac{1}{k} \int_{t_{j}}^{t_{j+1}} A(s) v d s\right\|_{V^{*}} \leq \frac{1}{k} \int_{t_{j}}^{t_{j+1}}\|A(s) v\|_{V^{*}} d s \\
& \leq \frac{1}{k} \int_{t_{j}}^{t_{j+1}} M\|v\|_{V} d s=M\|v\|_{V^{\prime}}
\end{aligned}
$$


with $j=0,1, \ldots, n-1$, and (2) is proved.

For (3), we have

$$
\begin{aligned}
\sum_{j=0}^{n-1}\left\|\bar{f}_{k_{j, j+1}}\right\|_{V^{*}}^{2} k & =\sum_{j=0}^{n-1}\left\|\frac{1}{k} \int_{t_{j}}^{t_{j+1}} f(s) d s\right\|_{V^{*}}^{2} k \leq \sum_{j=0}^{n-1} \frac{1}{k} \int_{t_{j}}^{t_{j+1}}\|f(s)\|_{V^{*}}^{2} d s k \\
& =\int_{0}^{T}\|f(s)\|_{V^{*}}^{2} d s \leq N,
\end{aligned}
$$

using Jensen's inequality and owing to (3) in Assumption 1.

As an immediate consequence of Proposition 1, the existence and uniqueness and the stability results, Theorems 2 and 3, respectively, hold for this particular scheme under Assumption 1 instead of Assumption 2. For the scheme's convergence, we state a new result.

Theorem 7. Let Assumption 1 be satisfied and assume that constant $K$ satisfies: $2 K k<$ 1. Denote by $u(t)$ the unique solution of problem (3) in Theorem 1. Assume that $A_{k}$ and $f_{k}$ in problem (5) are specified, respectively, by $\bar{A}_{k}$ and $\hat{f}_{k}$ in (39) and denote by $v_{k, j}, j=0$, $1, \ldots, n$, the unique solution of problem (5) in Theorem 2. Let Assumption 3 be satisfied. Then there exists a constant $N$ independent of $k$ such that

$$
\max _{0 \leq j \leq n}\left\|v_{k, j}-u\left(t_{j}\right)\right\|_{H}^{2} \leq N k^{2 \delta} \quad \text { and } \quad \sum_{j=0}^{n}\left\|v_{k, j}-u\left(t_{j}\right)\right\|_{V}^{2} k \leq N k^{2 \delta} .
$$

Proof. The estimates in Theorem 4 are obtained as an immediate consequence of Proposition 1. Additionally, due to the particular form of operator $\bar{A}_{k}$ and function $\hat{f}_{k}$, we have

$$
\begin{aligned}
& \sum_{j=1}^{n} \frac{1}{k}\left\|\bar{A}_{k, j} u\left(t_{j}\right) k-\int_{t_{j-1}}^{t_{j}} A(s) u\left(t_{j}\right) d s\right\|_{V^{*}}^{2} \\
& =\sum_{j=1}^{n} \frac{1}{k}\left\|\frac{1}{k} \int_{t_{j-1}}^{t_{j}} A(s) u\left(t_{j}\right) d s k-\int_{t_{j-1}}^{t_{j}} A(s) u\left(t_{j}\right) d s\right\|_{V^{*}}^{2}=0
\end{aligned}
$$

and

$$
\sum_{j=1}^{n} \frac{1}{k}\left\|\bar{f}_{k, j} k-\int_{t_{j-1}}^{t_{j}} f(s) d s\right\|_{V^{*}}^{2}=\sum_{j=1}^{n} \frac{1}{k}\left\|\frac{1}{k} \int_{t_{j-1}}^{t_{j}} f(s) d s k-\int_{t_{j-1}}^{t_{j}} f(s) d s\right\|_{V^{*}}^{2}=0 .
$$

The result follows.

From Theorem 7, we see that the rate of convergence is optimal when $A$ and $f$ are approximated by the integral averages $\bar{A}_{k}$ and $\hat{f}_{k}$, respectively.

Moreover, it can be easily checked that any operator $\hat{A} k$ and function $\hat{f}_{k}$ optimizing the rate of convergence coincide with $\bar{A}_{k}$ and $\hat{f}_{k}$, in the sense that 


$$
\left\|\hat{A}_{k, j+1} z-\bar{A}_{k, j+1} z\right\|_{V^{*}}=0 \text { and }\left\|\hat{f}_{k, j+1}-\bar{f}_{k, j+1}\right\|_{V^{*}}=0
$$

for all $z \in V, j=0,1, \ldots, n-1$. In fact,

$$
\begin{aligned}
k^{2}\left\|\hat{A}_{k, j} u\left(t_{j}\right)-\hat{A}_{k, j} u\left(t_{j}\right)\right\|_{V^{*}}^{2} \leq 2 & \left\|\hat{A}_{k, j} u\left(t_{j}\right) k-\int_{t_{j-1}}^{t_{j}} A(s) u\left(t_{j}\right) d s\right\|_{V^{*}}^{2} \\
& +2\left\|A_{k, j} u\left(t_{j}\right) k-\int_{t_{j-1}}^{t_{j}} A(s) u\left(t_{j}\right) d s\right\|_{V^{*}}^{2}=0
\end{aligned}
$$

and

$$
k^{2}\left\|\hat{f}_{k, j}-\bar{f}_{k, j}\right\|_{V^{*}}^{2} \leq 2\left\|\hat{f}_{k, j} k-\int_{t_{j-1}}^{t_{j}} f(s) d s\right\|_{V^{*}}^{2}+2\left\|\bar{f}_{k, j} k-\int_{t_{j-1}}^{t_{j}} f(s) d s\right\|_{V^{*}}^{2}
$$

for all $j=1,2, \ldots, n$.

Next, we investigate a different type of specification for $A_{k}$ and $f_{k}$ in problem (5).

Consider the pairs of discrete weight functions

$$
\left(\rho^{j+1}\left(t_{j}\right), \rho^{j+1}\left(t_{j+1}\right)\right),\left(r^{j+1}\left(t_{j}\right), r^{j+1}\left(t_{j+1}\right)\right), \quad j=0,1, \ldots, n-1
$$

such that

$$
\rho^{j+1}\left(t_{j}\right), \rho^{j+1}\left(t_{j+1}\right), r^{j+1}\left(t_{j}\right), r^{j+1}\left(t_{j+1}\right) \geq 0
$$

and

$$
\rho^{j+1}\left(t_{j}\right)+\rho^{j+1}\left(t_{j+1}\right)=r^{j+1}\left(t_{j}\right)+r^{j+1}\left(t_{j+1}\right)=1,
$$

for all $j=0,1, \ldots, n-1$.

We define the discrete operator

$$
\tilde{A}_{k}\left(t_{j+1}\right) z:=\rho^{j+1}\left(t_{j}\right) A\left(t_{j}\right) z+\rho^{j+1}\left(t_{j+1}\right) A\left(t_{j+1}\right) z
$$

and the discrete function

$$
\tilde{f}_{k}\left(t_{j+1}\right):=r^{j+1}\left(t_{j}\right) f\left(t_{j}\right)+r^{j+1}\left(t_{j+1}\right) f\left(t_{j+1}\right),
$$

for all $z \in V, j=0,1, \ldots, n-1$. Denote

$$
\tilde{A}_{k, j+1} z=\tilde{A}_{k}\left(t_{j+1}\right) z, \quad \tilde{f}_{k, j+1}=\tilde{f}_{k}\left(t_{j+1}\right)
$$

and

$$
\rho_{j}^{j+1}=\rho^{j+1}\left(t_{j}\right), \quad \rho_{j+1}^{j+1}=\rho^{j+1}\left(t_{j+1}\right), \quad r_{j}^{j+1}=r^{j+1}\left(t_{j}\right), \quad r_{j+1}^{j+1}=r^{j+1}\left(t_{j+1}\right),
$$

for all $z \in V, j=0,1, \ldots, n-1$.

We prove that, in this particular case, under Assumption 1, Assumption 2 is satisfied. 
Proposition 2. Under Assumption 1, $\tilde{A}_{k}$ and $\hat{f}_{k}$ satisfy

$$
\begin{aligned}
& \text { 1. }\left\langle\bar{A}_{k, j+1} v, v\right\rangle+\lambda\|v\|_{V}^{2} \leq K\|v\|_{H}^{2}, \forall v \in V, j=0,1, \ldots, n-1, \\
& \text { 2. }\left\|\bar{A}_{k, j+1} v\right\|_{V^{*}} \leq M\|v\|_{V}, \forall v \in V, j=0,1, \ldots, n-1, \\
& \text { 3. } \sum_{j=0}^{n-1}\left\|\bar{f}_{k, j+1}\right\|_{V^{*}}^{2} k \leq N,
\end{aligned}
$$

where $\lambda, K, M$, and $N$ are constants, with $\lambda, K$, and $M$ the constants in Assumption 1. Proof. For all $v \in V$, owing to (1) in Assumption 1,

$$
\begin{aligned}
\left\langle\tilde{A}_{k, j+1} v, v\right\rangle & =\left\langle\rho_{j}^{j+1} A\left(t_{j}\right) v+\rho_{j+1}^{j+1} A\left(t_{j+1}\right) v, v\right\rangle \\
& =\rho_{j}^{j+1}\left\langle A\left(t_{j}\right) v, v\right\rangle+\rho_{j+1}^{j+1}\left\langle A\left(t_{j+1}\right) v, v\right\rangle \\
& \leq\left(\rho_{j}^{j+1}+\rho_{j+1}^{j+1}\right)\left(K\|v\|_{H}^{2}-\lambda\|v\|_{V}^{2}\right)=K\|v\|_{H}^{2}-\lambda\|v\|_{V}^{2},
\end{aligned}
$$

with $j=0,1, \ldots, n-1$, and (1) is proved.

For all $v \in V$, owing to (2) in Assumption 1, we have

$$
\begin{aligned}
\left\|\tilde{A}_{k, j+1} v\right\|_{V *} & =\left\|\rho_{j}^{j+1} A\left(t_{j}\right) v+\rho_{j+1}^{j+1} A\left(t_{j+1}\right) v\right\|_{V *} \\
& \leq \rho_{j}^{j+1}\left\|A\left(t_{j}\right) v\right\|_{V *}+\rho_{j+1}^{j+1}\left\|A\left(t_{j+1}\right) v\right\|_{V *} \\
& \leq M\left(\rho_{j}^{j+1}+\rho_{j+1}^{j+1}\right)\|v\|_{V}=M\|v\|_{V}
\end{aligned}
$$

with $j=0,1, \ldots, n-1$, and (2) is proved.

Inequality (3) is satisfied trivially and the result is proved.

For this particular scheme, the existence and uniqueness and the stability results, respectively, Theorems 2 and 3, hold under Assumption 1 instead of Assumption 2 as an immediate consequence of Proposition 2.

In order to prove a result on the scheme's convergence, we assume further smoothness. Denote by $B\left(V, V^{*}\right)$ the Banach space of all bounded linear operators from $V$ into $V^{*}$. Also, denote by $\operatorname{Lip}([0, T] ; X)$ the space of Lipschitz-continuous $X$-valued functions on $[0, T]$, with $X$ a Banach space. Let both spaces be endowed with the usual norms.

\section{Assumption 6. Suppose that}

$$
\begin{aligned}
& \text { 1. } A \in \operatorname{Lip}\left([0, T] ; B\left(V, V^{*}\right)\right) \text {; } \\
& \text { 2. } f \in \operatorname{Lip}\left([0, T] ; V^{*}\right) .
\end{aligned}
$$

Remark 9. (1) and (2) in Assumption 6 could be replaced, respectively, by the weaker conditions

$$
A \in C^{\alpha}\left([0, T] ; B\left(V, V^{*}\right)\right) \text { and } f \in C^{\alpha}\left([0, T] ; V^{*}\right),
$$

where $0<\delta \leq \alpha \leq 1$, with $\delta$ the constant defined in Assumption 3.

Theorem 8. Let Assumption 1 be satisfied and assume further that constant $K$ satisfies: $2 K k<1$. Denote by $u(t)$ the unique solution of problem (3) in Theorem 1. Assume that $A_{k}$ and $f_{\mathrm{k}}$ in problem (5) are specified, respectively, by $\tilde{A}_{k}$ and $\tilde{f}_{k}$ in (40), (41) and denote by $v_{k, j}, j=0,1, \ldots, n$, the unique solution of problem (5) in Theorem 2. Let Assumptions 3 and 6 are satisfied. Then there exists a constant $N$ independent of $k$ 
such that

$$
\max _{0 \leq j \leq n}\left\|v_{k, j}-u\left(t_{j}\right)\right\|_{H}^{2} \leq N k^{2 \delta} \quad \text { and } \quad \sum_{j=0}^{n}\left\|v_{k, j}-u\left(t_{j}\right)\right\|_{V}^{2} k \leq N k^{2 \delta} .
$$

Proof. The estimates in Theorem 4 are obtained as an immediate consequence of Proposition 2. Due to the particular form of operator $\tilde{A}_{k}$ and function $\tilde{f}_{k}$, we have

$$
\begin{aligned}
& \sum_{j=1}^{n} \frac{1}{k}\left\|\tilde{A}_{k, j} u\left(t_{j}\right) k-\int_{t_{j-1}}^{t_{j}} A(s) u\left(t_{j}\right) d s\right\|_{V^{*}}^{2} \\
& \quad=\sum_{j=1}^{n} \frac{1}{k}\left\|\left(\rho_{j-1}^{j} A\left(t_{j-1}\right) u\left(t_{j}\right)+\rho_{j}^{j} A\left(t_{j}\right) u\left(t_{j}\right)\right) k-\int_{t_{j-1}}^{t_{j}} A(s) u\left(t_{j}\right) d s\right\|_{V^{*}}^{2} \\
& \quad=\sum_{j=1}^{n} \frac{1}{k}\left\|\int_{t_{j-1}}^{t_{j}}\left(\rho_{j-1}^{j} A\left(t_{j-1}\right)+\rho_{j}^{j} A\left(t_{j}\right)-\left(\rho_{j-1}^{j}+\rho_{j}^{j}\right) A(s)\right) u\left(t_{j}\right) d s\right\|_{V^{*}}^{2} \\
& \quad \leq \sum_{j=1}^{n} \int_{t_{j-1}}^{t_{j}}\left\|\left(\rho_{j-1}^{j}\left(A\left(t_{j-1}\right)-A(s)\right)+\rho_{j}^{j}\left(A\left(t_{j}\right)-A(s)\right)\right) u\left(t_{j}\right)\right\|_{V^{*}}^{2} d s,
\end{aligned}
$$

with the inequality obtained by the use of Jensen's inequality.

For the argument of the integral in (42),

$$
\begin{aligned}
& \left\|\left(\rho_{j-1}^{j}\left(A\left(t_{j-1}\right)-A(s)\right)+\rho_{j}^{j}\left(A\left(t_{j}\right)-A(s)\right)\right) u\left(t_{j}\right)\right\|_{V^{*}}^{2} \\
& \leq 2\left(\rho_{j-1}^{j}\right)^{2}\left\|\left(A\left(t_{j-1}\right)-A(s)\right) u\left(t_{j}\right)\right\|_{V^{*}}^{2}+2\left(\rho_{j}^{j}\right)^{2}\left\|\left(A\left(t_{j}\right)-A(s)\right) u\left(t_{j}\right)\right\|_{V^{*}}^{2} \\
& \leq 2\left(N\left|t_{j-1}-s\right| \cdot\left\|u\left(t_{j}\right)\right\|_{V}\right)^{2}+2\left(N\left|t_{j}-s\right| \cdot\left\|u\left(t_{j}\right)\right\|_{V}\right)^{2} \\
& \leq N k^{2}\left\|u\left(t_{j}\right)\right\|_{V}^{2} \leq N k^{2} \leq N k^{2 \delta},
\end{aligned}
$$

owing to (1) in Assumption 6.

Finally, from (42), (43),

$$
\sum_{j=1}^{n} \frac{1}{k}\left\|\tilde{A}_{k, j} u\left(t_{j}\right) k-\int_{t_{j-1}}^{t_{j}} A(s) u\left(t_{j}\right) d s\right\|_{V^{*}}^{2} \leq N \sum_{j=1}^{n} \int_{t_{j-1}}^{t_{j}} k^{2 \delta} d s \leq N k^{2 \delta} .
$$

Following the same steps, owing to (2) in Assumption 6, we also obtain

$$
\sum_{j=1}^{n} \frac{1}{k}\left\|\tilde{f}_{k, j} k-\int_{t_{j-1}}^{t_{j}} f(s) d s\right\|_{V^{*}}^{2} \leq N k^{2 \delta}
$$

and the result follows.

Remark 10. For $j=1, \ldots, n$, the two-point closed Newton-Cotes quadrature formulas for the integrals 


$$
\int_{t_{j-1}}^{t_{j}} A(s) u\left(t_{j}\right) d s \text { and } \int_{t_{j-1}}^{t_{j}} f(s) d s
$$

are written, respectively,

$$
\int_{t_{j-1}}^{t_{j}} A(s) u\left(t_{j}\right) d s \simeq \tilde{A}_{k, j} u\left(t_{j}\right) k \text { and } \int_{t_{j-1}}^{t_{j}} f(s) d s \simeq \tilde{f}_{k, j} k_{,}
$$

with the weights

$$
\left(\rho^{j}\left(t_{j-1}\right), \rho^{j}\left(t_{j}\right)\right)=\left(r^{j}\left(t_{j-1}\right), r^{j}\left(t_{j}\right)\right)=\left(\frac{1}{2}, \frac{1}{2}\right) .
$$

It can be easily shown that in the particular case where $A(s) u\left(t_{j}\right)$ and $f(s)$, with $t_{j-i} \leq s$ $\leq t_{j}$ and $j=1, \ldots, n$, are real-valued polynomials of degree 1 the approximation error is null.

\section{Examples for the explicit scheme}

In this section, we investigate the same types of specification for the discretised operator $A_{h k}$ and function $f_{h k}$ but now under the framework of the explicit scheme.

We begin by considering the particular case, where $A_{h k}$ and $f_{h k}$ in problem (19) are specified, respectively, by the integral averages

$$
\bar{A}_{h k}\left(t_{j}\right) z:=\frac{1}{k} \int_{t_{j}}^{t_{j+1}} A_{h}(s) z d s \text { and } \bar{f}_{h k}\left(t_{j}\right):=\frac{1}{k} \int_{t_{j}}^{t_{j+1}} f_{h}(s) d s,
$$

for all $z \in V_{h}, j=0,1, \ldots, n-1$. For all $z \in V_{h}$, denote

$$
\bar{A}_{h k, j} z=\bar{A}_{h k}\left(t_{j}\right) z, \quad \bar{f}_{h k, j}=\bar{f}_{h k}\left(t_{j}\right), \quad j=0,1, \ldots, n-1 .
$$

For this particular scheme, under Assumption 1, Assumption 4 is satisfied.

Proposition 3. Under Assumption 1, operator $\bar{A}_{h k}$ and function $\bar{f}_{h k}$ satisfy

$$
\begin{aligned}
& \text { 1. }\left\langle\bar{A}_{h k, j} v, v\right\rangle_{h}+\lambda\|v\|_{V_{h}}^{2} \leq K\|v\|_{H_{h}}^{2}, \forall v \in V_{h}, j=0,1, \ldots, n-1 \text {, } \\
& \text { 2. }\left\|\bar{A}_{h k, j} v\right\|_{V_{h}^{*}} \leq M\|v\|_{V_{h}}, \forall v \in V_{h}, j=0,1, \ldots, n-1, \\
& \text { 3. } \sum_{j=0}^{n-1}\left\|\bar{f}_{h k, j}\right\|_{V_{h}^{*}}^{2} k \leq N,
\end{aligned}
$$

where $\lambda, K, M$, and $N$ are the constants in Assumption 1.

Proof. Operator $\bar{A}_{h k, j}$ and function $\bar{f}_{h k, j}$ coincide, respectively, with $\bar{A}_{k, j+1}$ and $\bar{f}_{k, j+1}$ in (39), for $j=0,1, \ldots, n-1$, replacing $A$ and $\mathrm{f}$ by their versions $A_{h}$ and $f_{h}$ in the integrals' arguments. The result follows from Proposition 1.

Owing to Proposition 3, the stability result, Theorem 5, holds for this particular scheme under Assumption 1 instead of Assumption 4. 
As for the implicit scheme, an optimal rate of convergence is obtained when $A_{h}$ and $f_{h}$ are discretised, respectively, by the integral averages $\bar{A}_{h k}$ and $\bar{f}_{h k}$. The proof is the same as for Theorem 7.

Theorem 9. Let Assumptions 1 and 5 be satisfied, and $\lambda, K, M$, and $C_{h}$ the constants there defined. Denote by $u_{h}(t)$ the unique solution of problem (18) in Theorem 1. Assume that $A_{h k}$ and $f_{h k}$ in problem (19) are specified, respectively, by $\bar{A}_{h k}$ and $\bar{f}_{h k}$ in (44) and denote by $v_{h k, j}$, with $j=0,1, \ldots, n$, the unique solution of problem (19). Assume that constant $K$ is such that $2 K k<1$ and that Assumption 3 is satisfied. If there exists a number $p$ such that $M^{2} C_{h}^{2} k \leq p<\lambda$ then there exists a constant $N$, independent of $k$ and $h$, such that

$$
\max _{0 \leq j \leq n}\left\|v_{h k, j}-u_{h}\left(t_{j}\right)\right\|_{H_{h}}^{2} \leq N k^{2 \delta} \quad \text { and } \quad \sum_{j=0}^{n-1}\left\|v_{h k, j}-u_{h}\left(t_{j}\right)\right\|_{V_{h}}^{2} k \leq N k^{2 \delta} .
$$

Similarly to what we have done in Section 5, we study an alternative discretisation for $A_{h}$ and $f_{h}$ in problem (18). Consider the pairs of discrete weight functions

$$
\left(\rho^{j}\left(t_{j}\right), \rho^{j}\left(t_{j+1}\right)\right),\left(r^{j}\left(t_{j}\right), r^{j}\left(t_{j+1}\right)\right), \quad j=0,1, \ldots, n-1
$$

such that

$$
\rho^{j}\left(t_{j}\right), \rho^{j}\left(t_{j+1}\right), r^{j}\left(t_{j}\right), r^{j}\left(t_{j+1}\right) \geq 0
$$

and

$$
\rho^{j}\left(t_{j}\right)+\rho^{j}\left(t_{j+1}\right)=r^{j}\left(t_{j}\right)+r^{j}\left(t_{j+1}\right)=1,
$$

for all $j=0,1, \ldots, n-1$.

We define the discrete operator

$$
\tilde{A}_{h k}\left(t_{j}\right) z:=\rho^{j}\left(t_{j}\right) A_{h}\left(t_{j}\right) z+\rho^{j}\left(t_{j+1}\right) A_{h}\left(t_{j+1}\right) z
$$

and the discrete function

$$
\tilde{f}_{h k}\left(t_{j}\right):=r^{j}\left(t_{j}\right) f_{h}\left(t_{j}\right)+r^{j}\left(t_{j+1}\right) f_{h}\left(t_{j+1}\right),
$$

for all $z \in V, j=0,1, \ldots, n-1$. We denote

$$
\tilde{A}_{h k, j} z=\tilde{A}_{h k}\left(t_{j}\right) z, \quad \tilde{f}_{h k, j}=\tilde{f}_{h k}\left(t_{j}\right)
$$

and

$$
\rho_{j}^{j}=\rho^{j}\left(t_{j}\right), \quad \rho_{j+1}^{j}=\rho^{j}\left(t_{j+1}\right), \quad r_{j}^{j}=r^{j}\left(t_{j}\right), \quad r_{j+1}^{j}=r^{j}\left(t_{j+1}\right),
$$

for all $z \in V, j=0,1, \ldots, n-1$.

We prove that, under Assumption 1, Assumption 4 is satisfied.

Proposition 4. Under Assumption 1, $\tilde{A}_{h k}$ and $\tilde{f}_{h k}$ satisfy

$$
\begin{aligned}
& \text { 1. }\left\langle\tilde{A}_{h k, j} v, v\right\rangle_{h}+\lambda\|v\|_{V_{h}}^{2} \leq K\|v\|_{H_{h}}^{2}, \forall v \in V_{h}, j=0,1, \ldots, n-1, \\
& \text { 2. }\left\|\tilde{A}_{h k, j} v\right\|_{V_{h}^{*}} \leq M\|v\|_{V_{h}}, \forall v \in V_{h}, j=0,1, \ldots, n-1,
\end{aligned}
$$


3. $\sum_{j=0}^{n-1}\left\|\tilde{f}_{h k, j}\right\|_{V_{h}^{*}}^{2} k \leq N$,

where $\lambda, K, M$, and $N$ are constants, with $\lambda, K$, and $M$ the constants in Assumption 1. Proof. Operator $\tilde{A}_{h k, j}$ and function $\tilde{f}_{h k, j}$ coincide, respectively, with $\tilde{A}_{k, j+1}$ and $\tilde{f}_{k, j+1}$ in (40), (41), for $j=0,1, \ldots, n-1$, after replacing $A$ and $f$ by $A_{h}$ and $f_{h}$ in their analytic expressions. The result follows from Proposition 2.

Owing to Proposition 4, Theorem 5 on the scheme's stability holds under Assumption 1 instead of Assumption 4.

Finally, we state a result on the scheme's convergence. The proof is the same as for Theorem 8.

Theorem 10. Let Assumptions 1 and 5 be satisfied, and $\lambda, K, M$, and $C_{h}$ the constants there defined. Denote by $u_{h}(t)$ the unique solution of problem (18) in Theorem 3. Assume that $A_{h k}$ and $f_{h k}$ in problem (19) are specified, respectively, by $\tilde{A}_{h k}$ and $\tilde{f}_{h k}$ in (45), (46) and denote by $v_{h k, j}, j=0,1, \ldots, n$, the unique solution of problem (19). Assume that constant $K$ is such that $2 K k<1$ and that Assumptions 3 and 6 are satisfied. If there exists a number $p$ such that $M^{2} C_{h}^{2} k \leq p<\lambda$ then there exists a constant $N$, independent of $k$ and $h$, such that

$$
\max _{0 \leq j \leq n}\left\|v_{h k, j}-u_{h}\left(t_{j}\right)\right\|_{H_{h}}^{2} \leq N k^{2 \delta} \quad \text { and } \quad \sum_{j=0}^{n-1}\left\|v_{h k_{, j}}-u_{h}\left(t_{j}\right)\right\|_{V_{h}}^{2} k \leq N k^{2 \delta} .
$$

Remark 11. Remark 10 still applies here with the obvious modifications.

\section{Computational results}

In this final Section we apply the above study to the discretisation of the Cauchy problem for a linear parabolic PDE. When the implicit scheme is used, a previous localization procedure is needed. We avoid additional issues and discretise the PDE problem by using only an explicit scheme.

Let us consider problem (2) under the assumption that the PDE does not degenerate, and in the particular 1D case

$$
\frac{\partial u}{\partial t}=L u+f \text { in }[0, T] \times \mathbb{R}, \quad u(0, x)=g(x) \text { in } \mathbb{R},
$$

where $L$ is the second-order partial differential operator

$$
L(t, x)=a(t, x) \frac{\partial^{2}}{\partial x^{2}}+b(t, x) \frac{\partial}{\partial x}+c(t, x)
$$

with real-valued coefficient functions, and $f$ and $g$ given real-valued functions.

To make the framework simpler, we specify the PDE coefficients as bounded functions

$$
a(t, x)=\frac{10+\cos ^{2}(x)}{1+t}, \quad b(t, x)=\frac{\sin (x)}{1+t}, \quad \text { and } \quad c(t, x)=\frac{\cos (x)}{1+t}
$$


as well as the free data $f$ and $g$

$$
f(t, x)=\frac{1}{(1+t) \sqrt{1+x^{2}}}, \quad \text { and } \quad g(x)=\frac{1}{\sqrt{4+x^{2}}} .
$$

Then, problem (47) reads

$$
\frac{\partial u}{\partial t}=L u+\frac{1}{(1+t) \sqrt{1+x^{2}}} \text { in }[0, T] \times \mathbb{R}, \quad u(0, x)=\frac{1}{\sqrt{4+x^{2}}} \text { in } \mathbb{R},
$$

where

$$
L(t, x)=\frac{10+\cos ^{2}(x)}{1+t} \frac{\partial^{2}}{\partial x^{2}}+\frac{\sin (x)}{1+t} \frac{\partial}{\partial x}+\frac{\cos (x)}{1+t} .
$$

We now semi-discretise problem (48) in the spatial variable. Consider the $h$-grid $Z_{h}$ on $\mathbb{R}$, with $h \in(0,1]$,

$$
Z_{h}=\{x \in \mathbb{R}: x=n h, \quad n=0, \pm 1, \pm 2, \ldots\},
$$

For every $x \in Z_{h}$, with $w$ a real valued function in $[0, T] \times Z_{h}$, denote

$$
\partial^{+} w=\partial^{+} w(t, x)=h^{-1}(w(t, x+h)-w(t, x))
$$

and

$$
\partial^{-} w=\partial^{-} w(t, x)=h^{-1}(w(t, x)-w(t, x-h)),
$$

the forward and backward difference quotients in space, respectively. For $t \in[0, T]$ and $x \in Z_{h}$, define the operator

$$
L_{h}(t, x)=a(t, x) \partial^{-} \partial^{+}+b(t, x) \partial^{+}+c(t, x)
$$

and the data functions

$$
f_{h}(t, x):=f(t, x)=\frac{1}{(1+t) \sqrt{1+x^{2}}} \quad \text { and } \quad g_{h}(x):=g(x)=\frac{1}{\sqrt{4+x^{2}}},
$$

discrete versions of $L, f$, and $g$, respectively.

We then have the following space-discretised version of problem (48)

$$
\frac{\partial u}{\partial t}=L_{h} u+\frac{1}{(1+t) \sqrt{1+x^{2}}} \text { in }[0, T] \times Z_{h,} \quad u(0, x)=\frac{1}{\sqrt{4+x^{2}}} \text { in } Z_{h},
$$

where

$$
L_{h}(t, x)=\frac{10+\cos ^{2}(x)}{1+t} \partial^{-} \partial^{+}+\frac{\sin (x)}{1+t} \partial^{+}+\frac{\cos (x)}{1+t} .
$$

By considering problem (48) in the appropriate classes of Sobolev spaces and problem (49) in their discrete versions, it can be shown that both problems (48) and (49) can be cast into problem (3). Therefore, problems (48) and (49) have unique generalized solutions. Moreover, the solution of the space-discretised problem (49) converges to the solution of the exact problem (48). The theoretical justification of the above is beyond the scope of the present study. For this, we refer to [2-4], where the spatial 
approximation of a more general PDE problem is investigated by using standard variational techniques.

Finally, we fully discretise problem (48) by discretising problem (49) in the time variable $t$. Consider the $n$-grid $T_{n}$ on $[0, T]$ in (4). We use the explicit scheme (19) and obtain

$$
\begin{cases}\Delta^{+} v\left(t_{i}, x\right)=L_{h k}\left(t_{i}, x\right) v\left(t_{i}, x\right)+f_{h k}\left(t_{i}, x\right), & i=0,1 \ldots, n-1, x \in Z_{h} \\ v(0, x)=\frac{1}{\sqrt{4+x^{2}}}, & x \in Z_{h} .\end{cases}
$$

Operator $L_{h k}$ and function $f_{h k}$ will be specified considering the two alternative procedures studied in Section 6:

- by using integral averages,

$$
\begin{aligned}
& \bar{L}_{h k}\left(t_{i}, x\right) \\
& =\frac{1}{k} \int_{t_{i}}^{t_{i+1}} \frac{10+\cos ^{2}(x)}{1+s} d s \partial^{-} \partial^{+}+\frac{1}{k} \int_{t_{i}}^{t_{i+1}} \frac{\sin (x)}{1+s} d s \partial^{+}+\frac{1}{k} \int_{t_{i}}^{t_{i+1}} \frac{\cos (x)}{1+s} d s
\end{aligned}
$$

and

$$
\bar{f}_{h k}\left(t_{i}, x\right)=\frac{1}{k} \int_{t_{i}}^{t_{i+1}} \frac{1}{(1+s) \sqrt{1+x^{2}}} d s,
$$

for all $i=0,1, \ldots, n-1$;

- and by using arithmetic averages, with weights

$$
\begin{aligned}
\left(\rho^{i}\left(t_{i}\right), \rho^{i}\left(t_{i+1}\right)\right)= & \left(r^{i}\left(t_{i}\right), r^{i}\left(t_{i+1}\right)\right)=(1 / 2,1 / 2) \text { for } i=0,1, \ldots, n-1, \\
\tilde{L}_{h k}\left(t_{i}, x\right)= & \frac{1}{2}\left(\frac{10+\cos ^{2}(x)}{1+t_{i}} \partial^{-} \partial^{+}+\frac{\sin (x)}{1+t_{i}} \partial^{+}+\frac{\cos (x)}{1+t_{i}}\right) \\
& +\frac{1}{2}\left(\frac{10+\cos ^{2}(x)}{1+t_{i+1}} \partial^{-} \partial^{+}+\frac{\sin (x)}{1+t_{i+1}} \partial^{+}+\frac{\cos (x)}{1+t_{i+1}}\right)
\end{aligned}
$$

and

$$
\tilde{f}_{h k}\left(t_{i, x} x\right)=\frac{1}{2} \cdot \frac{1}{\left(1+t_{i}\right) \sqrt{1+x^{2}}}+\frac{1}{2} \cdot \frac{1}{\left(1+t_{i+1}\right) \sqrt{1+x^{2}}},
$$

for all $i=0,1, \ldots, n-1$.

Let us consider the stepsizes $h=1$ and $k=0.01$ for problem (50). It can be easily checked that the hypotheses of Theorems 5, 9, and 10 hold. Therefore the schemes are stable and the solution $v_{h k}$ of the fully discretised problem (50) converges to the solution $u_{h}$ of the semi-discretised problem (49). Both numerical schemes were implemented making use of the software Mathematica, version 8.0.4. We considered the intervals $[0,10]$ and $[-20,20]$ for the time and space variables, respectively.

In Figures 1 and 2, there is a representation of the solution $v_{h k}$ of problem (50): Figure 1 corresponds to the integral average type of discretisation and Figure 2 to the arithmetic average type. 


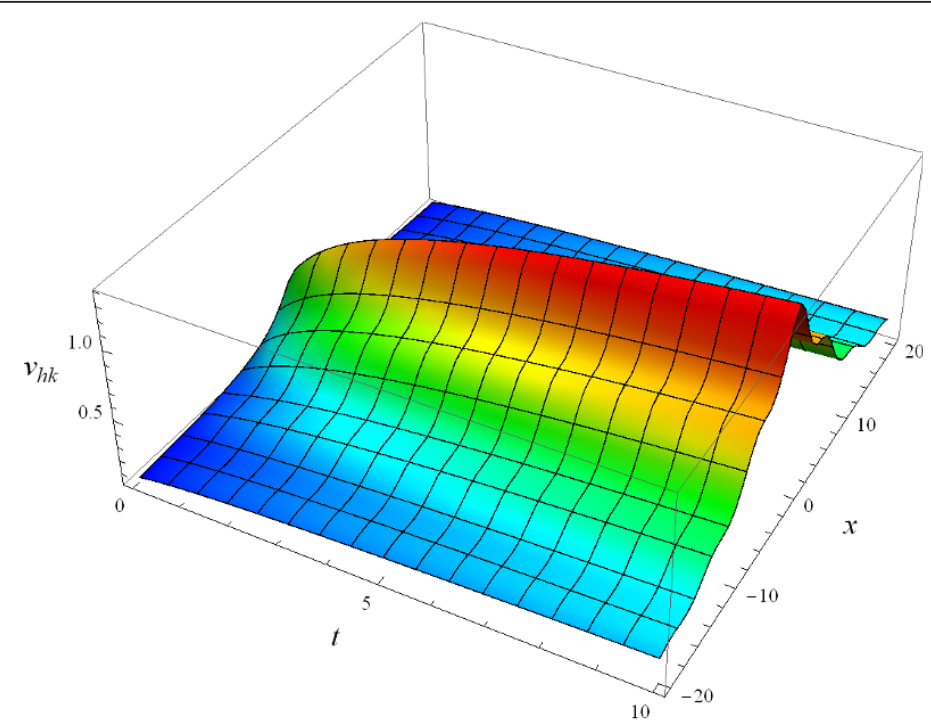

Figure 1 The solution $v_{h k}$ determined with the integral average explicit scheme, with $T=10, h=1$, and $k=0.01$.

We note that the solution surfaces are very similar. In fact, we have

$$
\max _{t_{j}, x}\left|\frac{v_{h k}^{1}\left(t_{j}, x\right)-v_{h k}^{2}\left(t_{j}, x\right)}{v_{h k}^{1}\left(t_{j}, x\right)}\right| \simeq 5.13035 .10^{-6},
$$

where $v_{h k}^{1}$ and $v_{h k}^{2}$ denote the solutions of problem (50) obtained, respectively, with the integral average and the arithmetic average types of discretisation.

This implies that, for this particular example, the arithmetic average discretisation produces a solution very close to the solution obtained with the optimal integral average discretisation.

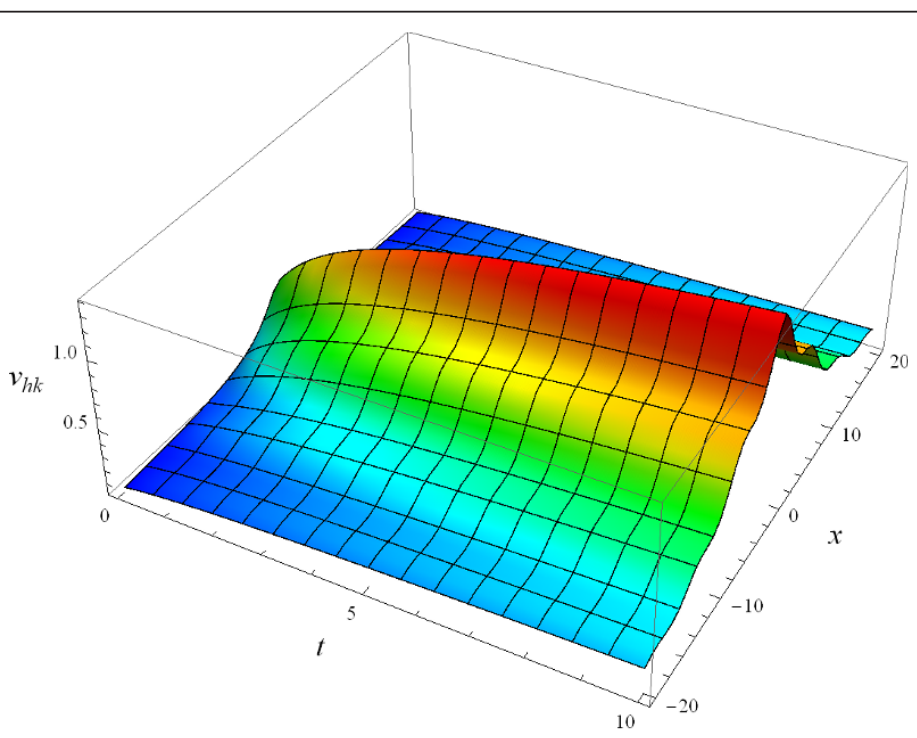

Figure 2 The solution $v_{h k}$ determined with the arithmetic average explicit scheme, with $T=10, h=$ 1 , and $k=0.01$. 


\section{Acknowledgements}

Fundação para a Ciência e Tecnologia - FCT (SFRH/BPD/35734/2008) to FF Gonçalves. Fundação para a Ciência e Tecnologia - FCT (FEDER/POCI 2010) to MR Grosssinho. Fundação para a Ciência e Tecnologia - FCT (SFRH/BD/42160/ 2007) to E Morais.

\section{Author details}

${ }^{1}$ CEMAPRE, ISEG - Technical University of Lisbon, Rua do Quelhas 6, 1200-781 Lisboa, Portugal ${ }^{2}$ Department of Mathematics, ISEG - Technical University of Lisbon, Rua do Quelhas 6, 1200-781 Lisboa, Portugal ${ }^{3}$ Department of Mathematics, University of Trás-os-Montes e Alto Douro, Apartado 1013, 5001-801 Vila Real, Portugal

\section{Authors' contributions}

The manuscript was elaborated jointly by the authors, in its several stages. Therefore there is no special mention concerning the authors' contributions. All authors read and approved the final manuscript.

\section{Competing interests}

The authors declare that they have no competing interests.

Received: 6 October 2011 Accepted: 16 February 2012 Published: 16 February 2012

\section{References}

1. Lamberton, D, Lapeyre, B: Introduction to Stochastic Calculus Applied to Finance. Chapman and Hall, UK (1996)

2. Gonçalves, FF, Grossinho, MR, Morais, E: On the space discretization of PDEs with unbounded coefficients arising in Financial Mathematics - the case of one spatial dimension. C R Acad Bulg Sci. 63(1):35-46 (2010)

3. Gonçalves, FF, Grossinho, MR: Space discretization of Cauchy problems for multidimensional PDEs with unbounded coefficients arising in Financial Mathematics. C R Acad Bulg Sci. 62(7):791-798 (2009)

4. Gonçalves, FF: Numerical approximation of partial differential equations arising in financial option pricing. PhD thesis, University of Edinburgh, School of Mathematics http://cemapre.iseg.utl.pt/ fernando/fernando_thesis.pdf (2007)

5. Brenner, Ph, Thomée, V: On rational approximations of semigroups. SIAM J Numer Anal. 16, 683-694 (1979). doi:10.1137/0716051

6. Crouzeix, M, Larsson, S, Piskarevv, S, Thomée, V: The stability of rational approximations of analytic semigroups. BIT. 33 74-84 (1993). doi:10.1007/BF01990345

7. Crouzeix, M: On multistep approximation of semigroups in Banach spaces. J Comput Appl Math. 20, 25-36 (1987)

8. Le Roux, M-N: Semidiscretization in time for parabolic problems. Math Comp. 33(147):919-931 (1979)

9. Lenferink, HWJ, Spijker, MN: On the use of stability regions in the numerical analysis of initial value problems. Math Comp. 57, 221-237 (1991). doi:10.1090/50025-5718-1991-1079026-6

10. Bakaev, NYu: On variable stepsize Runge-Kutta approximations of a Cauchy problem for the evolution equation. BIT. 38(3):462-485 (1998). doi:10.1007/BF02510254

11. González, C, Palencia, C: Stability of Runge-Kutta methods for abstract time-dependent parabolic problems: the Hölder case. Math Comput. 68(225):73-89 (1999). doi:10.1090/S0025-5718-99-01018-2

12. González, C, Palencia, C: Stability of time-stepping methods for abstract time-dependent parabolic problems. SIAM J Numer Anal. 35(3):973-989 (1998). doi:10.1137/S0036142995283412

13. Palencia, C: A stability result for sectorial operators in Banach spaces. SIAM J Numer Anal. 30, 1373-1384 (1993). doi:10.1137/0730071

14. Gianazza, U, Savaré, G: Abstract evolution equations on variable domains: an approach by minimizing movements. Ann Scuola Norm Sup Pisa Cl Sci (5). 23(1):149-178 (1996)

15. Gyöngy, I, Millet, A: Rate of convergence of space time approximations for stochastic evolution equations. Potential Anal. 30(1):29-64 (2009). doi:10.1007/s11118-008-9105-5

16. Lions, JL, Magenes, E: Problèmes aux Limites Non Homogènes et Applications, vol. 2 (in French). Dunod, GauthierVillars, Paris, France (1968)

17. Lions, JL: Quelques Méthodes de Résolution des Problèmes aux Limites Non Linéaires (in French). Dunod, GauthierVillars, Paris, France (1969)

doi:10.1186/1687-1847-2012-14

Cite this article as: Gonçalves et al: Discretisation of abstract linear evolution equations of parabolic type.

Advances in Difference Equations 2012 2012:14. 